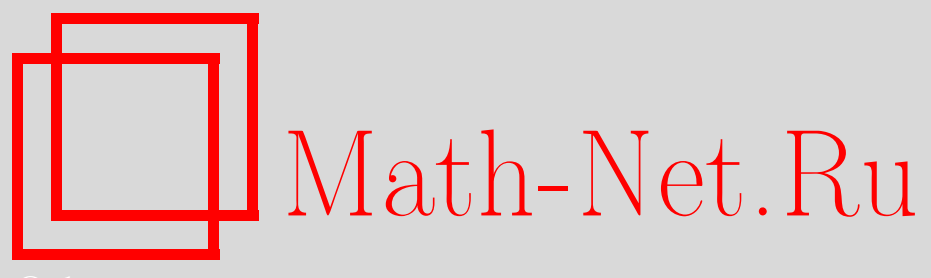

Е. В. Степанова, А. Е. Шишков, Начальная эволюция носителей решений квазилинейных параболических уравнений с вырождающимся абсорбционным потенциалом, Матем. сб., 2013, том 204, номер 3, 79-106

DOI: https://doi.org/10.4213/sm8121

Использование Общероссийского математического портала Math-Net.Ru подразумевает, что вы прочитали и согласны с пользовательским соглашением http://www . mathnet.ru/rus/agreement

Параметры загрузки:

IP : 54.162 .85 .209

26 апреля 2023 г., 15:54:10

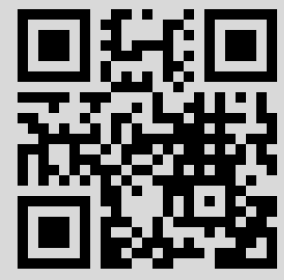


Е. В. Степанова, А. Е. Шишков

\section{Начальная эволюция носителей решений квазилинейных параболических уравнений с вырождающимся абсорбционным потенциалом}

В работе изучено распространение носителей решений квазилинейных параболических уравнений второго порядка типа нестационарной диффузии - полулинейной абсорбции с вырождающимся на начальной плоскости абсорбционным потенциалом. Найдены в определенном смысле точные достаточные условия на соотношение граничного режима и характер вырождения потенциала, которые гарантируют сильную локализацию решений, а также установлена ослабленная локализация решений при произвольном потенциале, который вырождается только на начальной плоскости.

Библиография: 12 названий.

Ключевые слова: квазилинейные параболические уравнения, абсорбционный потенциал, сильная локализация решений, ослабленная локализация решений, энергетический метод.

DOI: $10.4213 / \mathrm{sm} 8121$

\section{$\S 1$. Постановка задачи и формулировка результатов}

1.1. Постановка задачи. Пусть $\Omega \subset\left\{x \in \mathbb{R}^{n}:|x|>1\right\}$ - ограниченная область в $\mathbb{R}^{n}\left(n \geqslant 1, \operatorname{diam} \Omega=L_{1}\right)$ с липшицевой границей $\partial \Omega=\partial_{0} \Omega \cup \partial_{1} \Omega$, где

$$
\partial_{0} \Omega=\left\{x \in \mathbb{R}^{n}:|x|=1\right\}, \quad \partial_{1} \Omega \subset\left\{x \in \mathbb{R}^{n}:|x|>l\right\}, \quad l=\text { const }>1 .
$$

В цилиндрической области $Q_{T}=(0, T) \times \Omega, 0<T<\infty$, рассматривается следующая задача Коши-Дирихле:

$$
\begin{gathered}
u_{t}-\sum_{i=1}^{n}\left(a_{i}\left(t, x, u, \nabla_{x} u\right)\right)_{x_{i}}+g(t, x)|u|^{q-1} u=0 \quad \text { в } \quad Q_{T}, \quad 0<q<1, \\
u(t, x)=f(t, x) \quad \text { на } \begin{array}{c}
(0, T) \times \partial_{0} \Omega, \\
u(0, x)=0 \quad \forall x \in \Omega .
\end{array}
\end{gathered}
$$

Здесь непрерывные по совокупности аргументов функции $a_{i}(t, x, s, \xi), i=1$, $\ldots, n$, при всех $(t, x, s, \xi) \in(0, T) \times \Omega \times \mathbb{R} \times \mathbb{R}^{n}$ удовлетворяют следующим условиям:

$$
\begin{gathered}
\left|a_{i}(t, x, s, \xi)\right| \leqslant d_{1}|\xi|, \quad d_{1}=\text { const }<\infty \\
\sum_{i=1}^{n}\left(a_{i}(t, x, s, \xi)-a_{i}(t, x, s, \eta)\right)\left(\xi_{i}-\eta_{i}\right) \geqslant d_{0}|\xi-\eta|^{2}, \quad d_{0}=\text { const }>0
\end{gathered}
$$


а непрерывная неотрицательная функция $g(t, x)$ вырождается при $t=0$, т.е.

$$
g(t, x)>0 \quad \forall(t, x) \in(0, T] \times \bar{\Omega}, \quad g(0, x)=0 \quad \forall x \in \bar{\Omega} .
$$

Существование энергетического (слабого) решения (см. определение 1) задачи $(1.1)-(1.7)$ при естественных предположениях на режим $f(t)$ (в частности, при ограниченности функции $F(t)$, строящейся по $f(t))$ следует из результатов работы [1].

Хорошо известно (см., например, [2], [3] и имеющиеся там ссылки), что любое обобщенное решение задачи (1.1)-(1.4) в случае невырождения абсорбционного потенциала $g(t, x)$, т.е. при условии

$$
g(t, x) \geqslant c_{0}>0 \quad \forall(t, x) \in(0, T] \times \bar{\Omega},
$$

обладает свойством конечности скорости распространения носителя. Отсюда, в частности, следует локализованность носителя решения

$$
\zeta(t):=\sup \{|x|: x \in \operatorname{supp} u(t, \cdot)\}<c_{1}=c_{1}\left(T_{1}\right)<l \quad \forall t: 0 \leqslant t<T_{1}=T_{1}(l) \leqslant T .
$$

Эффект локализации носителей решений различных классов квазилинейных и полулинейных параболических уравнений изучался во многих работах (см., например, [2], [4], где можно найти дальнейшие ссылки). А. С. Калашниковым в [5] в случае одномерного полулинейного уравнения теплопроводности $(n=1$, $\left.a_{i}(t, x, s, \xi)=\xi, \xi \in \mathbb{R}\right)$ с вырождающимся абсорбционным потенциалом $g_{0}(t) \in$ $C^{1}([0,+\infty)) \cap L_{\infty}([0,+\infty)), g_{0}(0)=0, g_{0}(t)>0$ для любого $t>0$, и граничным режимом $u(t, 1)=f(t) \in C^{1}([0,+\infty)) \cap L_{\infty}([0,+\infty))$ было установлено свойство ослабленной локализации

$$
\sup \{\zeta(t): 0<\delta \leqslant t<T\}<c_{1}=c_{1}(\delta)<\infty \quad \forall \delta>0
$$

при выполнении следующего условия подчиненности граничного режима абсорбционному потенциалу:

$$
g_{0}(t)^{-1} \cdot f(t) \rightarrow 0 \quad \text { при } t \rightarrow 0 .
$$

С другой стороны, следуя гипотезе Г. И. Баренблатта о возможности начального скачка свободной границы $\zeta(t)$ в нестационарных краевых задачах, А. С. Калашников в работе [5] доказал свойство

$$
\inf \left\{\zeta(t): 0<t<t_{*}\right\} \geqslant c_{2}=c_{2}\left(t_{*}\right)>0
$$

для класса достаточно быстро убывающих при $t \rightarrow 0$ потенциалов $g_{0}(t)$. В частности, скачок (1.12) имеет место в случае следующего потенциала и граничного режима:

$$
g_{0}(t)=t^{1 / 2} \exp \left(-\frac{1}{t^{2}}\right), \quad f_{0}(t)=t \exp \left(-\frac{1}{t^{2}}\right) .
$$

Подчеркнем, что анализ в [5] касается только случая сильно вырождающихся граничных режимов $f(t)$ (см. условие (1.11)). Отметим также, что имеющиеся в [5] результаты получены барьерной техникой. Построение же аналогичных барьеров в данной ситуации авторам статьи не представляется возможным. 
В настоящей работе изучается эволюция носителей решений задачи (1.1)-(1.4) с произвольными граничными режимами $f(t, x)$ (как с сильно или слабо вырождающимися, так и с невырождающимися при $t \rightarrow 0)$ и с вырождающимся при $t=0$ потенциалом $g(t, x)$. Метод исследования основан на получении подходящих локальных интегральных априорных оценок решений в окрестности начальной плоскости $t=0$ и связан с комбинацией идей и построений из метода локальных энергетических оценок (см. [3], [6]), априорных оценок типа принципа Сен-Венана (см. [7]). Подходящие версии этого метода при изучении различных других качественных свойств обобщенных решений квазилинейных и полулинейных параболических уравнений с вырождающимся абсорбционным потенциалом использовались ранее в [8], [9]. В настоящей работе устанавливаются в определенном смысле точные достаточные условия сильной локализации (непрерывность $\zeta(t)$ при $t=0)$ носителя, а также ослабленная локализация решений при произвольном потенциале, вырождающемся только при $t=0$.

1.2. Формулировка результатов. Для произвольного множества $\Gamma \subset \partial \Omega$ через $H^{1}(\Omega, \Gamma):=W_{2}^{1}(\Omega, \Gamma)$ обозначаем, как обычно, замыкание в норме соболевского пространства $W_{2}^{1}(\Omega)$ множества функций из $C^{\infty}(\Omega)$, обращающихся в нуль в окрестности $\Gamma$, а через $\langle\cdot, \cdot\rangle$ - операцию спаривания элементов пространств $H^{1}(\Omega, \Gamma)$ и $\left(H^{1}(\Omega, \Gamma)\right)^{*}$. Без ограничения общности будем полагать, что функция $f(t, x)$ из граничного условия (1.3) определена на всей области $(0, T) \times \Omega$, причем

$$
f(t, \cdot) \in L_{2}\left(0, T ; H^{1}\left(\Omega, \partial_{1} \Omega\right)\right) \cap H^{1}\left(0, T ; L_{2}(\Omega)\right) .
$$

ОПРЕДЕЛЕНИЕ 1. Следуя [1], энергетическим (слабъм) решением задачи (1.1)-(1.4) называем функцию

$$
u(t, \cdot) \in f(t, \cdot)+L_{2}\left(0, T ; H^{1}(\Omega, \partial \Omega)\right)
$$

такую, что

$$
u_{t}(t, \cdot) \in L_{2}\left(0, T ;\left(H^{1}(\Omega, \partial \Omega)\right)^{*}\right)
$$

(и, следовательно, в силу [10] $u(t, x) \in C\left(0, T ; L_{2}(\Omega)\right)$, справедливо интегральное тождество

$$
\begin{aligned}
\int_{(0, T)}\left\langle u_{t}, \xi\right\rangle d t+\int_{(0, T) \times \Omega} \sum_{i=1}^{n} a_{i}\left(t, x, u, \nabla_{x} u\right) \xi_{x_{i}} d x d t \\
\quad+\int_{(0, T) \times \Omega} g(t, x)|u|^{q-1} u \xi d x d t=0 \quad \forall \xi \in L_{2}\left(0, T ; H^{1}(\Omega, \partial \Omega)\right)
\end{aligned}
$$

и выполняется начальное условие (1.4).

Отметим еще раз, что при сделанных предположениях задача (1.1)-(1.4) в силу [1] имеет решение $u(t, x)$ из определения 1 . 
Введем теперь следующую функцию $F(t)$, которая будет моделировать граничный режим $f(t, x)$ из (1.3) и фигурировать во всех наших дальнейших формулировках:

$$
\begin{aligned}
F(t):= & \sup _{0 \leqslant s \leqslant t} \int_{\Omega} f(s, x)^{2} d x+\int_{0}^{t} \int_{\Omega}\left(\left|\nabla_{x} f\right|^{2}+g(t, x)|f(t, x)|^{q+1}\right) d x d t \\
& +\int_{0}^{t} \int_{\Omega}\left|f_{t}(t, x)\right|^{2} d x d t .
\end{aligned}
$$

Для произвольного $t>0$ через $\zeta(t)$ будем, как и выше, обозначать определяемый в (1.9) радиус компактификации носителя рассматриваемого энергетического (слабого) решения задачи (1.1)-(1.4).

ТЕОРЕма 1 (о сильной локализации). Пусть абсорбционный потенциал $g(t, x)$ из (1.2) обладает неотрицательной монотонной минорантой

$$
g(t, x) \geqslant g_{0}(t)>0 \quad \forall t>0, \quad g_{0}(0)=0 .
$$

Пусть функция $F(\cdot)$ из (1.18), моделирующая граничный режим, удовлетворяет следующему условию подчиненности миноранте $g_{0}$ : для произвольного $t: 0<t \leqslant T$, существует $S=S(t)>0$ такое, что

$$
\begin{gathered}
F(t)^{(1-\psi)(1-q) / 2}<S^{2}\left(\int_{\tau}^{t} g_{0}(t)^{1-\theta} d t\right)^{2}+D_{1} \int_{0}^{\tau} g_{0}(t)^{2(1-\theta)} d t:=G(S, t, \tau) \\
\forall \tau \in(0, t),
\end{gathered}
$$

причем

$$
t S(t) \longrightarrow 0 \quad \text { npu } t \longrightarrow 0 \text {. }
$$

Здесъ $0<D_{1}=D_{1}\left(n, q, d_{0}, d_{1}, 2 l\right)=$ const $<\infty, l$ uз $(1.1)$,

$$
0<\theta:=\frac{(q+1)+n(1-q)}{2(q+1)+n(1-q)}<1, \quad 0<\psi:=\frac{n(1-q)}{2(q+1)+n(1-q)}<\theta<1 .
$$

Тогда рассматриваемое энергетическое решение и $(t, x)$ задачи (1.1)-(1.4) обладает свойством сильной (строгой) локализации и для радиуса компактификации носителя решения справедлива следующая оченка сверху:

$$
\zeta(t) \leqslant 1+\operatorname{ct} S(t)
$$

ЗАмечАнИЕ 1 . На протяжении этой работы через $C, c, c_{i}, i=0, \ldots, 16$, мы обозначаем различные положительные постоянные, которые зависят лишь от известных параметров задачи (1.1)-(1.4) $n, q, d_{0}, d_{1}$.

Приведем теперь несколько простых достаточных условий выполнения (1.20), (1.21).

СЛЕДСТВИЕ 1. Пусть абсорбиионный потенииал $g$ из (1.2) удовлетворяет условию (1.19), а функиия $F(\cdot)$ из (1.18), моделирующая граничньй режим, удовлетворяет соотношению

$$
F(t)^{(1-\psi)(1-q) / 2}<\frac{D_{1}}{2 t}\left(\int_{0}^{t} g_{0}(\tau)^{1-\theta} d \tau\right)^{2} \quad \forall t: 0<t \leqslant T_{1}=\frac{l-1}{c S\left(T_{1}\right)},
$$


где $0<D_{1}=D_{1}\left(n, q, d_{0}, d_{1}, 2 l\right)=$ const $<\infty, l$ из (1.1), $S$ из теоремь $1, \psi$ из $(1.22)$.

Тогда имеет место сильная локализация и справедлива следующая оценка для радиуса компактификации носителя решения:

$$
\zeta(t) \leqslant 1+c \sqrt{D_{1} t} \quad \forall t \leqslant T_{1} .
$$

СлЕДствие 2 (случай “умеренно” вырождающихся потенциалов). Пусть миноранта gо из (1.19) обладает дополнительным свойством

$$
\begin{gathered}
g_{0}(t) \geqslant g_{0, \beta}(t):=\exp \left(-\frac{\omega_{0}}{t^{\beta}}\right) \\
\forall t: 0<t \leqslant T_{1}=\frac{l-1}{c S\left(T_{1}\right)}, \quad \omega_{0}=\text { const }>0, \quad \beta \in(0,1),
\end{gathered}
$$

а функиия $F(\cdot)$ из (1.18), моделирующая граничный режим, удовлетворяет соотношению

$$
F(t)^{(1-\psi)(1-q) / 2}<\frac{D_{2}}{2 t^{1+\beta}}\left(\int_{0}^{t} g_{0, \beta}^{1-\theta}(\tau) d \tau\right)^{2} \quad \forall t: 0<t \leqslant T_{1},
$$

где $0<D_{2}=D_{2}\left(n, q, d_{0}, d_{1}, 2 l, \theta, \beta, \omega_{0}\right)=$ const $<\infty, l$ из (1.1), $S$ из теоремъ 1 , $\psi$ и $ө$ из (1.22).

Тогда имеет место сильная локализация и справедлива следующая оценка для радиуса компактификачии носителя решения:

$$
\zeta(t) \leqslant 1+c \sqrt{D_{2} t^{1-\beta}} \quad \forall t \leqslant T_{1} .
$$

СлЕДствиЕ 3 (случай “сильно” вырождающихся потенциалов). Пусть миноранта gо из (1.19) обладает дополнительным свойством

$$
\begin{gathered}
g_{0}(t) \geqslant g_{0, \beta}(t):=\exp \left(-\frac{\omega_{0}}{t^{\beta}}\right) \\
\forall t: 0<t \leqslant T_{1}=\frac{l-1}{c S\left(T_{1}\right)}, \quad \omega_{0}=\text { const }>0, \quad \beta \geqslant 1,
\end{gathered}
$$

l из (1.1), S из теоремы 1, а функиия $F(\cdot)$ из (1.18), моделирующая граничныи режим, удовлетворяет неравенству

$$
F(t)^{(1-\psi)(1-q) / 2}<\frac{\mu^{2}(t)}{t^{2}}\left(\int_{0}^{t} g_{0, \beta}^{1-\theta}(\tau) d \tau\right)^{2} \quad \forall t: 0<t \leqslant T_{1},
$$

где $\mu(\tau)>0: \mu(\tau) \rightarrow 0$ при $\tau \rightarrow 0 ;$ постоянные $\psi$ и $\theta$ из (1.22).

Тогда имеет место сильная локализация и справедлива следующая оченка для радиуса компактификации носителя решения:

$$
\zeta(t) \leqslant 1+c \mu(t) \quad \forall t \leqslant T_{1} .
$$

ЗАмЕчАНИЕ 2. Из теоремы 3, приведенной ниже, следует, что для “умеренно" в смысле (1.26) вырождающихся потенциалов $g$ строгая локализация имеет место при произвольных граничных режимах, в том числе и не удовлетворяющих никаким условиям подчиненности типа (1.20). Для различных же классов сильно вырождающихся потенциалов такие условия подчиненности могут быть обязательными (см. свойства (1.12), (1.13) из [5]). 
ТЕОРЕма 2 (об ослабленной локализации при произвольном граничном режиме). Пусть абсорбиионный потенциал g удовлетворяет условию (1.7).

Тогда для произвольного энергетического решения $u(t, x)$ задачи (1.1)-(1.4) имеет место свойство ослабленной локализации, т.е. существует непрерывная при $t>0$, не зависящая от $L_{1}$ функиия $\zeta_{1}(t)<\infty$ для любого $t>0$ (которая может стремиться $\kappa$ бесконечности при $t \rightarrow 0$ ) такая, что имеет место

$$
\zeta(t) \leqslant \min \left(\zeta_{1}(t), c L_{1}\right) \quad \forall t>0
$$

где $\zeta(\cdot)$ из (1.9), $c=$ const $>0, L_{1}=$ const $=\operatorname{diam} \Omega$.

ЗАмЕчАНИЕ 3 . Фраза в формулировке теоремы 2 “... $\zeta_{1}(t)$ может стремиться к бесконечности при $t \rightarrow 0$ " подчеркивает тот факт, что не исключается возможность бесконечного скачка, поэтому и речь в данной теореме идет лишь об ослабленной локализации. Из доказательства теоремы 2 следует, что в зависимости от функции $F(\cdot)$ из (1.18), моделирующей граничный режим, и миноранты абсорбционного потенциала $g_{0}$ возможны следующие варианты:

1) $\zeta_{1}(t) \rightarrow 1 \quad$ при $t \rightarrow 0$,

2) $\zeta_{1}(t) \rightarrow 1+$ const $\quad$ при $t \rightarrow 0$,

3) $\zeta_{1}(t) \rightarrow \infty$ при $t \rightarrow 0$.

ТЕОРЕМА 3 (о сильной локализации при произвольном граничном режиме). Пусть функиия $F(\cdot)$ из (1.18) моделирует граничный режим, а абсорбиионньй потенциал g из (1.2) обладает неотрицательной монотонной миноранmoù

$$
g(t, x) \geqslant g_{\omega}(t):=\exp \left(-\frac{\omega(t)}{t}\right) \quad \forall t>0,
$$

где $\omega(t)$ - произвольная неотрицательная неубывающая функиия: $\omega(t) \rightarrow 0$ npu $t \rightarrow 0$.

Тогда произвольное энергетическое решение и задачи (1.1)-(1.4) обладает свойством сильной (строгой) локализации и для радиуса компактификации носителя решения справедлива следующая оченка сверху:

$$
\zeta(t) \leqslant 1+\frac{t}{2}+C\left\{t \ln \left(C_{1} F(t)\right)+c_{1} t \ln t^{-1}+c_{2} \omega\left(\frac{t}{2}\right)\right\}^{1 / 2} \quad \forall t<T .
$$

ЗАмечАниЕ 4. В теоремах 2 и 3 нет никаких условий на граничный режим, т.е. на функцию $F(\cdot)$ из $(1.18)$.

\section{§ 2. Условия сильной (строгой) локализации при произвольных вырождающихся абсорбционных потенциалах}

В настоящем параграфе проводится доказательство теоремы 1 и ее следствий.

2.1. Вспомогательные построения и утверждения. Сначала установим глобальную энергетическую оценку произвольного решения $u(t, x)$ рассматриваемой задачи (1.1)-(1.4). 
Лемма 1. Для произвольных $\tau \leqslant T$ справедлива следующая интегральная априорная оценка:

$$
\sup _{0 \leqslant t \leqslant \tau} \int_{\Omega} u(t, x)^{2} d x+\int_{0}^{\tau} \int_{\Omega}\left(d_{0}\left|\nabla_{x} u\right|^{2}+g(t, x)|u(t, x)|^{q+1}\right) d x d t \leqslant C F(\tau),
$$

где функция $F(\cdot)$ из (1.18).

ДокАзАТЕльство. Для произвольного $\tau \in(0, T]$ определим пробную функцию следующим образом:

$$
\xi(t, x):=\xi_{\tau}(t, x)= \begin{cases}u(t, x)-f(t, x), & \text { если } t \leqslant \tau, \\ 0, & \text { если } t>\tau .\end{cases}
$$

Подстановка этой функции в тождество (1.17) дает

$$
\begin{gathered}
\int_{(0, \tau)}\left\langle u_{t}, u-f\right\rangle d t+\int_{(0, \tau) \times \Omega} \sum_{i=1}^{n} a_{i}\left(t, x, u, \nabla_{x} u\right)(u-f)_{x_{i}} d x d t \\
+\int_{(0, \tau) \times \Omega} g(t, x)|u|^{q-1} u(u-f) d x d t=0 .
\end{gathered}
$$

В силу формулы интегрирования по частям (см. $[1 ;$ пп. $1.5,1.6])$ имеем

$$
\begin{aligned}
\int_{(0, \tau)}\left\langle u_{t}, u\right. & -f\rangle d t=\frac{1}{2} \int_{\Omega} u(\tau, x)^{2} d x+\int_{0}^{\tau} \int_{\Omega} u(t, x) f_{t}(t, x) d x d t \\
& -\int_{\Omega} u(\tau, x) f(\tau, x) d x .
\end{aligned}
$$

Поэтому из (2.2) с учетом структурных условий (1.5) и (1.6) следует

$$
\begin{aligned}
\frac{1}{2} \int_{\Omega} u(\tau, x)^{2} d x & +\int_{0}^{\tau} \int_{\Omega}\left(d_{0}\left|\nabla_{x} u\right|^{2}+g(t, x)|u(x, t)|^{q+1}\right) d x d t \\
\leqslant & d_{1} \int_{0}^{\tau} \int_{\Omega}\left|\nabla_{x} u\right|\left|\nabla_{x} f(t, x)\right| d x d t+\int_{0}^{\tau} \int_{\Omega} g(t, x)|u(t, x)|^{q}|f(t, x)| d x d t \\
& +\int_{0}^{\tau} \int_{\Omega}|u(t, x)|\left|f_{t}(t, x)\right| d x d t+\int_{\Omega}|u(\tau, x)||f(\tau, x)| d x
\end{aligned}
$$

Оценивая сверху при помощи неравенств Юнга с $\varepsilon$ слагаемые справа в неравенстве (2.3), приходим после простых вычислений к

$$
\begin{aligned}
\int_{\Omega} u(\tau, x)^{2} d x & +\int_{0}^{\tau} \int_{\Omega}\left(d_{0}\left|\nabla_{x} u\right|^{2}+g(t, x)|u(t, x)|^{q+1}\right) d x d t \\
\leqslant & \frac{1}{2 T} \int_{0}^{\tau} \int_{\Omega} u(t, x)^{2} d x d t+c_{1} \int_{\Omega}|f(\tau, x)|^{2} d x \\
& +c_{2} \int_{0}^{\tau} \int_{\Omega}\left(\left|\nabla_{x} f(t, x)\right|^{2}+g(t, x)|f(t, x)|^{q+1}+\left|f_{t}(t, x)\right|^{2}\right) d x d t
\end{aligned}
$$

где $c_{1}=c_{1}\left(q, n, d_{0}, d_{1}\right)<\infty, c_{2}=c_{2}\left(q, n, d_{0}, d_{1}, T\right)<\infty$. Пусть теперь число $s \in[0, \tau]$ определено так, что

$$
\int_{\Omega} u(s, x)^{2} d x=\sup _{0 \leqslant t \leqslant \tau} \int_{\Omega} u(t, x)^{2} d x .
$$


В силу произвольности $\tau$ из (2.4) следует также

$$
\begin{aligned}
\int_{\Omega} u(s, x)^{2} d x & +\int_{0}^{s} \int_{\Omega}\left(d_{0}\left|\nabla_{x} u\right|^{2}+g(t, x)|u(t, x)|^{q+1}\right) d x d t \\
\leqslant & \frac{1}{2} \int_{\Omega}|u(s, x)|^{2} d x+c_{1} \int_{\Omega}|f(s, x)|^{2} d x \\
& \quad+c_{2} \int_{0}^{s} \int_{\Omega}\left(\left|\nabla_{x} f(t, x)\right|^{2}+g(t, x)|f(t, x)|^{q+1}+\left|f_{t}(t, x)\right|^{2}\right) d x d t .
\end{aligned}
$$

Комбинируя (2.4) и (2.6), приходим к неравенству

$$
\begin{aligned}
\sup _{0 \leqslant t \leqslant \tau} & \int_{\Omega}|u(t, x)|^{2} d x+\int_{0}^{\tau} \int_{\Omega}\left(d_{0}\left|\nabla_{x} u\right|^{2}+g(t, x)|u(t, x)|^{q+1}\right) d x d t \leqslant c_{3} F(\tau) \\
:= & c_{3}\left[\sup _{0 \leqslant s \leqslant \tau} \int_{\Omega} f(s, x)^{2} d x\right. \\
& \left.+\int_{0}^{\tau} \int_{\Omega}\left(\left|\nabla_{x} f\right|^{2}+g(t, x)|f(t, x)|^{q+1}+\left|f_{t}(t, x)\right|^{2}\right) d x d t\right],
\end{aligned}
$$

что и соответствует доказываемой оценке (2.1).

Введем теперь следующие семейства подобластей, связанных с областью $\Omega$ :

$$
\Omega(s):=\Omega \cap\left\{x \in \mathbb{R}^{n}:|x|>s\right\} \quad \forall s=\text { const }>1 .
$$

Лемма 2. При произвольных $0 \leqslant a<b \leqslant T$ и почти всех $s>1$ для произволъного энергетического решения $u(t, x)$ задачи (1.1)-(1.4) справедливо следующее соотношение:

$$
\begin{aligned}
& \frac{1}{2} \int_{\Omega(s)} u(b, x)^{2} d x+\int_{a}^{b} \int_{\Omega(s)}\left(\sum_{i=1}^{n} a_{i}\left(t, x, u, \nabla_{x} u\right) u_{x_{i}}+g(t, x)|u|^{q+1}\right) d x d t \\
& =\frac{1}{2} \int_{\Omega(s)} u(a, x)^{2} d x+\int_{a}^{b} \int_{\partial_{0} \Omega(s)} \sum_{i=1}^{n} a_{i}\left(t, x, u, \nabla_{x} u\right) u \nu_{i} d \sigma d t:=R_{0}+R_{1},
\end{aligned}
$$

где $\partial_{0} \Omega(s)=\partial \Omega(s) \cap\left\{x \in \mathbb{R}^{n}:|x|=s\right\}, \vec{\nu}=\vec{\nu}(x)=\left\{\nu_{i}\right\}-$ единичный вектор внешней нормали $\kappa \partial_{0} \Omega(s)$ в точке $x$.

ДокАЗАТЕЛЬСтво. Зафиксируем числа $s>1, \delta>0$ и введем липшицеву срезающую функцию $\eta_{s, \delta}(r)$ :

$$
\begin{gathered}
\eta_{s, \delta}(r)=0 \quad \forall r<s, \quad \eta_{s, \delta}(r)=1 \quad \forall r>s+\delta, \\
\eta_{s, \delta}(r)=\delta^{-1}(r-s) \quad \forall r: s<r<s+\delta .
\end{gathered}
$$

Подставим в интегральное тождество (1.17) пробную функцию

$$
\xi(t, x)= \begin{cases}u(t, x) \eta_{s, \delta}(|x|) & \forall t: a \leqslant t \leqslant b, \\ 0 & \forall t \subset\{t<a\} \cup\{t>b\} .\end{cases}
$$


В силу формулы интегрирования по частям имеем

$$
\begin{aligned}
\frac{1}{2} \int_{\Omega(s)} u(b, x)^{2} \eta_{s, \delta} d x+\int_{a}^{b} \int_{\Omega(s)}\left(\sum_{i=1}^{n} a_{i}\left(t, x, u, \nabla_{x} u\right) u_{x_{i}}+g(t, x)|u|^{q+1}\right) \eta_{s, \delta} d x d t \\
=\frac{1}{2} \int_{\Omega(s)} u(a, x)^{2} \eta_{s, \delta} d x \\
\quad-\int_{a}^{b} \int_{\Omega(s) \backslash \Omega(s+\delta)} \sum_{i=1}^{n} a_{i}\left(t, x, u, \nabla_{x} u\right) u\left(\eta_{s, \delta}(|x|)\right)_{x_{i}} d x d t .
\end{aligned}
$$

Переходя теперь в последнем равенстве к пределу при $\delta \rightarrow 0$ (как это было сделано в работе [3]), устанавливаем, что при почти всех $s$ существует

$$
\int_{a}^{b} \int_{\partial_{0} \Omega(s)} \sum_{i=1}^{n} a_{i}\left(t, x, u, \nabla_{x} u\right) u \nu_{i} d \sigma d t
$$

и справедливо соотношение (2.9).

Для дальнейшего изучения свойств решения задачи (1.1)-(1.4) установим два класса локальных энергетических оценок. Сначала установим оценки, ориентированные на абсорбционный член уравнения (1.2). С этой целью введем энергетические функции, связанные с рассматриваемым решением $и$ исходной задачи:

$$
\begin{gathered}
H_{\tau}(s):=\int_{\Omega(s)}|u(\tau, x)|^{2} d x \\
E_{\tau}(s):=\int_{\Omega(s)}\left(d_{0}\left|\nabla_{x} u(\tau, x)\right|^{2}+g_{0}(\tau)|u(\tau, x)|^{q+1}\right) d x \\
I_{\tau}^{b}(s):=\int_{\tau}^{b} \int_{\Omega(s)}\left(d_{0}\left|\nabla_{x} u(t, x)\right|^{2}+g_{0}(t)|u(t, x)|^{q+1}\right) d x d t,
\end{gathered}
$$

а также функцию

$$
J_{\tau}^{b}(s):=\int_{\tau}^{b} \int_{\partial_{0} \Omega(s)}\left|\nabla_{x} u(t, x)\right|^{2} d \sigma d t
$$

где $g_{0}(\cdot)$ - миноранта потенциала $g$, определяемая в (1.19).

ЗАмЕчаниЕ 5. Ясно, что в любом случае (при непрерывности изменения носителя $u(t, x)$ и при ее отсутствии) для функции $\zeta(t)$ из (1.9) выполняется неравенство

$$
\zeta(t) \leqslant L_{1}=\text { const }=\operatorname{diam} \Omega \quad \forall t>0 .
$$

При этом, очевидно, справедлива следующая оценка:

$$
\text { meas supp } u(t, \cdot) \leqslant c_{(n)} \min \left\{L_{1}^{n}, \zeta(t)^{n-1}(\zeta(t)-1)\right\}:=l(t),
$$

где $c_{(n)}$ - постоянная, зависящая только от $n$. 
Лемма 3. Функиии $H_{\tau}(\cdot), J_{\tau}^{b}(\cdot), I_{\tau}^{b}(\cdot)$, определеннъе в $(2.11)$, при всех $\tau \leqslant T$ и почти всех $s>1$ удовлетворяют следующему соотношению:

$$
\begin{aligned}
& H_{T}(s)+I_{\tau}^{T}(s) \\
& \quad \leqslant 2 H_{\tau}(s)+c_{1} L_{\tau}(T) g_{0}(\tau)^{-2(1-\theta) /(2-(1-\theta)(1-q))}\left[J_{\tau}^{T}(s)\right]^{2 /(2-(1-\theta)(1-q))},
\end{aligned}
$$

где $0<\theta<1$ из (1.22),

$$
L_{\tau}(T):=\sup _{\tau \leqslant t \leqslant T} l(t)^{(1-q)(1-q)(1-\theta) /((q+1)(2-(1-q)(1-\theta)))},
$$

$c_{1}=$ const $<\infty$ не зависит от $u(t, x)$, а бункиия $l(\cdot)$ из (2.13).

ДоКАЗАТЕЛЬСТВо. В силу того, что $u(t, x)=0$ на $\partial_{1} \Omega$, интерполяционное неравенство в теореме о следах функций из $W_{p}^{l}(\Omega)$ (в частности, из $H^{1}(\Omega)$ ) относительно функции $u$ принимает следующий вид (см., например, [3]):

$$
\begin{aligned}
& \left(\int_{\partial_{0} \Omega(s)}|u(t, x)|^{2} d \sigma\right)^{1 / 2} \\
& \quad \leqslant d_{3}\left(\int_{\Omega(s)}\left|\nabla_{x} u\right|^{2} d x\right)^{\theta / 2}\left(\int_{\Omega(s)}|u(t, x)|^{q+1} d x\right)^{(1-\theta) /(q+1)} \quad \forall t>0,
\end{aligned}
$$

где $\theta$ из $(1.22), d_{3}=d_{3}(n, q)=$ const. Применим неравенство (2.15) для оценки второго слагаемого в правой части соотношения (2.9). Используя дополнительно условие (1.5), а также неравенства Гёльдера и Юнга, получаем

$$
\begin{aligned}
\bar{R}_{1}(t):=\left|\int_{\partial_{0} \Omega(s)} \sum_{i=1}^{n} a_{i}\left(t, x, u, \nabla_{x} u\right) u \nu_{i} d \sigma\right| \\
\leqslant d_{1}\left(\int_{\partial_{0} \Omega(s)}\left|\nabla_{x} u(t, x)\right|^{2} d \sigma\right)^{1 / 2}\left(\int_{\partial_{0} \Omega(s)}|u(t, x)|^{2} d \sigma\right)^{1 / 2} \\
\leqslant d_{1} d_{3}\left(\int_{\partial_{0} \Omega(s)}\left|\nabla_{x} u\right|^{2} d \sigma\right)^{1 / 2}\left(\int_{\Omega(s)}\left|\nabla_{x} u\right|^{2} d x\right)^{\theta / 2}\left(\int_{\Omega(s)}|u|^{q+1} d x\right)^{(1-\theta) / 2} \\
\quad \times\left(\int_{\Omega(s)}|u|^{q+1} d x\right)^{(1-q)(1-\theta) /(2(q+1))} \\
\leqslant c d_{1} d_{3}\left(\int_{\partial_{0} \Omega(s)}\left|\nabla_{x} u\right|^{2} d \sigma\right)^{1 / 2}\left(\int_{\Omega(s)}\left|\nabla_{x} u\right|^{2}+g_{0}(t)|u|^{q+1} d x\right)^{1 / 2} \\
\quad \times g_{0}(t)^{-(1-\theta) / 2}\left(\int_{\Omega(s)}|u|^{q+1} d x\right)^{(1-q)(1-\theta) /(2(q+1))} .
\end{aligned}
$$

В силу неравенства Гёльдера и неравенства (2.13)

$$
\int_{\Omega(s)}|u(t, x)|^{q+1} d x \leqslant l(t)^{(1-q) / 2}\left(\int_{\Omega(s)}|u(t, x)|^{2} d x\right)^{(q+1) / 2},
$$


что после подстановки в (2.16) дает

$$
\begin{aligned}
\bar{R}_{1}(t) \leqslant & c d_{1} d_{3}\left(\int_{\partial_{0} \Omega(s)}\left|\nabla_{x} u\right|^{2} d \sigma\right)^{1 / 2} \\
& \times\left(\int_{\Omega(s)}\left(\left|\nabla_{x} u\right|^{2}+g_{0}(t)|u|^{q+1}\right) d x\right)^{1 / 2} g_{0}(t)^{-(1-\theta) / 2} \\
& \times l(t)^{(1-q)(1-q)(1-\theta) /(4(q+1))}\left(\int_{\Omega(s)}|u(t, x)|^{2} d x\right)^{(1-q)(1-\theta) / 4} \forall t>0 .
\end{aligned}
$$

Интегрируя (2.18) по $t$ и используя неравенство Юнга с $\varepsilon$, получаем

$$
\begin{aligned}
\left|R_{1}\right| \leqslant \varepsilon & \int_{a}^{b} \int_{\Omega(s)}\left(\left|\nabla_{x} u\right|^{2}+g_{0}(t)|u|^{q+1}\right) d x d t \\
& +c(\varepsilon) g_{0}(a)^{-(1-\theta)} l_{a}(b)^{(1-q)(1-q)(1-\theta) /(2(q+1))} \\
& \times\left(\int_{\Omega(s)}\left|u\left(b_{1}, x\right)\right|^{2} d x\right)^{(1-q)(1-\theta) / 2} \int_{a}^{b} \int_{\partial_{0} \Omega(s)}\left|\nabla_{x} u\right|^{2} d \sigma d t \quad \forall \varepsilon>0,
\end{aligned}
$$

где $l_{a}(b)=\sup _{a \leqslant t \leqslant b} l(t)$, функция $l(t)$ из $(2.13), R_{1}$ из $(2.9), a \leqslant b_{1}=b_{1}(a, b) \leqslant b$ таково, что

$$
\int_{\Omega(s)} u\left(b_{1}, x\right)^{2} d x=\max _{a \leqslant t \leqslant b} \int_{\Omega(s)} u(t, x)^{2} d x .
$$

Оценим теперь $\int_{\Omega(s)} u\left(b_{1}, x\right)^{2} d x$ сверху при помощи $(2.9)$ с $b=b_{1},(2.19)$ и неравенства Юнга с $\varepsilon_{1}$ :

$$
\begin{aligned}
\frac{1}{2} \int_{\Omega(s)} \mid & \left.u\left(b_{1}, x\right)\right|^{2} d x+\int_{a}^{b_{1}} \int_{\Omega(s)}\left(d_{0}\left|\nabla_{x} u\right|^{2}+g_{0}(t)|u|^{q+1}\right) d x d t \\
\leqslant & \frac{1}{2} \int_{\Omega(s)}|u(a, x)|^{2} d x+\varepsilon \int_{a}^{b_{1}} \int_{\Omega(s)}\left(d_{0}\left|\nabla_{x} u\right|^{2}+g_{0}(t)|u|^{q+1}\right) d x d t \\
& +c(\varepsilon) g_{0}(a)^{-(1-\theta)} l_{a}\left(b_{1}\right)^{(1-q)(1-q)(1-\theta) /(2(q+1))} \\
& \times\left(\int_{\Omega(s)}\left|u\left(b_{1}, x\right)\right|^{2} d x\right)^{(1-q)(1-\theta) / 2} \int_{a}^{b_{1}} \int_{\partial_{0} \Omega(s)}\left|\nabla_{x} u\right|^{2} d \sigma d t \\
\leqslant & \frac{1}{2} \int_{\Omega(s)}|u(a, x)|^{2} d x+\varepsilon \int_{a}^{b_{1}} \int_{\Omega(s)}\left(d_{0}\left|\nabla_{x} u\right|^{2}+g_{0}(t)|u|^{q+1}\right) d x d t \\
& +\varepsilon_{1} \int_{\Omega(s)}\left|u\left(b_{1}, x\right)\right|^{2} d x \\
& +c\left(\varepsilon_{1}\right)\left(g_{0}(a)^{-(1-\theta)} l_{a}\left(b_{1}\right)^{(1-q)(1-q)(1-\theta) /(2(q+1))}\right)^{2 /(2-(1-q)(1-\theta))} \\
& \times\left(\int_{a}^{b_{1}} \int_{\partial_{0} \Omega(s)}\left|\nabla_{x} u\right|^{2} d \sigma d t\right)^{2 /(2-(1-q)(1-\theta))}
\end{aligned}
$$


Полагая в последнем неравенстве $\varepsilon=1 / 2, \varepsilon_{1}=1 / 4$, получаем

$$
\begin{aligned}
\frac{1}{4} \int_{\Omega(s)} \mid & \left.u\left(b_{1}, x\right)\right|^{2} d x+\frac{1}{2} \int_{a}^{b_{1}} \int_{\Omega(s)}\left(d_{0}\left|\nabla_{x} u\right|^{2}+g_{0}(t)|u|^{q+1}\right) d x d t \\
\leqslant \frac{1}{2} & \int_{\Omega(s)}|u(a, x)|^{2} d x+c_{3} g_{0}(a)^{-2(1-\theta) /(2-(1-q)(1-\theta))} \\
& \times l_{a}\left(b_{1}\right)^{(1-q)(1-q)(1-\theta) /((q+1)(2-(1-q)(1-\theta)))} \\
& \times\left(\int_{a}^{b_{1}} \int_{\Omega(s)}\left|\nabla_{x} u\right|^{2} d \sigma d t\right)^{2 /(2-(1-q)(1-\theta))} .
\end{aligned}
$$

Отсюда при произвольных $s>1, \nu>0$ вытекает следующее соотношение для энергетических функций, введенных в (2.11):

$$
\begin{aligned}
{\left[H_{b_{1}}(s)\right]^{\nu} } & \leqslant c_{4}\left[H_{a}(s)\right]^{\nu} \\
& +c_{5} L_{a}\left(b_{1}\right)^{\nu} g_{0}(a)^{-2(1-\theta) \nu /(2-(1-\theta)(1-q))}\left[J_{a}^{b_{1}}(s)\right]^{2 \nu /(2-(1-\theta)(1-q))}
\end{aligned}
$$

где $c_{4}=c_{4}(\nu)=$ const $<\infty$ и $c_{5}=c_{5}(\nu)=$ const $<\infty$. Дополнительно из равенства (2.9) с учетом условия (1.6) и оценки (2.19) с $\varepsilon=d_{0} / 2$ вытекает соотношение

$$
\begin{aligned}
\frac{1}{2} H_{b}(s)+ & \frac{d_{0}}{2} I_{a}^{b}(s) \leqslant \frac{1}{2} H_{a}(s)+c_{6} L_{a}(b)^{(2-(1-\theta)(1-q)) / 2} g_{0}(a)^{-(1-\theta)} \\
& \times J_{a}^{b}(s)\left(H_{b_{1}}(s)\right)^{(1-q)(1-\theta) / 2}
\end{aligned}
$$

где $c_{6}=$ const $<\infty$. Оценим теперь последний сомножитель в правой части (2.22) при помощи неравенства $(2.21)$ с

$$
\nu=\frac{(1-q)(1-\theta)}{2} .
$$

В результате получаем

$$
\begin{aligned}
H_{b}(s)+ & I_{a}^{b}(s) \leqslant H_{a}(s)+c_{6} L_{a}(b)^{(2-(1-\theta)(1-q)) / 2} g_{0}(a)^{-(1-\theta)} J_{a}^{b}(s) \\
& \times\left[c_{4}\left[H_{a}(s)\right]^{\nu}+c_{5} L_{a}\left(b_{1}\right)^{\nu} g_{0}(a)^{-2(1-\theta) \nu /(2-(1-\theta)(1-q))}\right. \\
& \left.\times\left[J_{a}^{b_{1}}(s)\right]^{2 \nu /(2-(1-\theta)(1-q))}\right] \\
\leqslant & H_{a}(s)+c_{7} L_{a}(b)^{(2-(1-\theta)(1-q)) / 2} g_{0}(a)^{-(1-\theta)} J_{a}^{b}(s) H_{a}(s)^{\nu} \\
& +c_{8} L_{a}(b)^{\nu+(2-(1-\theta)(1-q)) / 2} g_{0}(a)^{-(1-\theta)(1+2 \nu /(2-(1-\theta)(1-q)))} \\
& \times\left[J_{a}^{b}(s)\right]^{1+2 \nu /(2-(1-\theta)(1-q))} .
\end{aligned}
$$

Оценивая второе слагаемое справа при помощи неравенства Юнга с $\varepsilon$, получаем после простых преобразований с использованием значения $\nu$ из $(2.23)$ необходимое соотношение (2.14) при $b=T, a=\tau$.

Следующая наша цель - оценить функцию $H_{\tau}(\cdot)$ в правой части $(2.14)$ некоторой функцией, связанной с основной энергетической функцией $I_{\tau}^{T}(\cdot)$. 
Лемма 4. Для функиий $H_{\tau}(\cdot), E_{\tau}(\cdot)$ из (2.11) при всех $\tau \leqslant T$ и почти всех $s>1$ справедливо неравенство

$$
\begin{aligned}
H_{\tau}(s) \leqslant & c_{2} g_{0}(\tau)^{-2(1-\psi) /(2-(1-\psi)(1-q))} \\
& \times l(\tau)^{(1-\psi)(1-q)(1-q) /((1+q)(2-(1-\psi)(1-q)))}\left[E_{\tau}(s)\right]^{2 /(2-(1-\psi)(1-q))}
\end{aligned}
$$

где $c_{2}=$ const $<\infty$ не зависит от $u(t, x)$, функиия $l(\cdot)$ из $(2.13)$, постоянная $\psi$ из (1.22).

ДокАЗАТЕльство. В силу интерполяционного неравенства Ниренберга-Гальярдо имеем

$$
\int_{\Omega(s)}|u(\tau, x)|^{2} d x \leqslant d_{4}\left(\int_{\Omega(s)}\left|\nabla_{x} u(\tau, x)\right|^{2} d x\right)^{\psi}\left(\int_{\Omega(s)}|u(\tau, x)|^{q+1} d x\right)^{2(1-\psi) /(q+1)},
$$

где $d_{4}=d_{4}(n, q)=\mathrm{const}, \psi$ из (1.22). Продолжая неравенство $(2.26)$, получаем

$$
\begin{aligned}
H_{\tau}(s) \leqslant d_{4} & \left(\int_{\Omega(s)}\left|\nabla_{x} u(\tau, x)\right|^{2} d x\right)^{\psi}\left(\int_{\Omega(s)} g_{0}(\tau)|u(\tau, x)|^{q+1} d x\right)^{1-\psi} \\
& \times g_{0}(\tau)^{-(1-\psi)}\left(\int_{\Omega(s)}|u(\tau, x)|^{q+1} d x\right)^{(1-\psi)(1-q) /(q+1)} \quad \forall s>1 .
\end{aligned}
$$

Из последнего неравенства (2.27), применяя неравенства Юнга, Гёльдера и соотношение (2.17), выводим

$$
H_{\tau}(s) \leqslant d_{4} g_{0}(\tau)^{-(1-\psi)} l(\tau)^{(1-\psi)(1-q)(1-q) /(2(1+q))} E_{\tau}(s)\left[H_{\tau}(s)\right]^{(1-\psi)(1-q) / 2} .
$$

Отсюда в силу неравенства Юнга непосредственно вытекает необходимое соотношение (2.25).

Лемма 5. Для функицй $H_{\tau}(\cdot), E_{\tau}(\cdot), I_{\tau}^{b}(\cdot), J_{\tau}^{b}(\cdot)$ из (2.11) справедливо следующее основное соотношение:

$$
\begin{aligned}
H_{T}(s)+ & I_{\tau}^{T}(s) \leqslant c_{0} g_{0}(\tau)^{-a(\psi)} l(\tau)^{b(\psi)} E_{\tau}(s)^{d(\psi)} \\
& +c_{1} g_{0}(\tau)^{-a(\theta)} l_{\tau}(T)^{c(\theta)}\left[J_{\tau}^{T}(s)\right]^{d(\theta)} \quad \forall s>1, \quad \forall \tau \leqslant T,
\end{aligned}
$$

где $l_{\tau}(T)=\sup _{\tau \leqslant t \leqslant T} l(t), l(t)$ из $(2.13)$, постоянные $\theta, \psi$ из $(1.22), c_{0}=$ const $<$ $\infty, c_{1}=$ const $<\infty$ не зависят от $u(t, x)$, а функиии $a(\cdot), b(\cdot), c(\cdot), d(\cdot)$ определяются следующим образом:

$$
\begin{aligned}
& a(\xi):=\frac{2(1-\xi)}{2-(1-\xi)(1-q)}, \quad b(\xi):=\frac{(1-\xi)(1-q)(1-q)}{(1+q)(2-(1-\xi)(1-q))}, \\
& c(\xi):=\frac{(q+1)(2-(1-q)(1-\xi))}{(1-q)(1-q)(1-\xi)}, \quad d(\xi):=\frac{2}{2-(1-\xi)(1-q)} .
\end{aligned}
$$

Для доказательства подставляем (2.25) в правую часть (2.14) и приходим к доказываемому соотношению (2.28). 
Проведем теперь некоторую трансформацию полученного основного энергетического соотношения (2.28). Будем далее полагать, что параметры $\tau, s$ в (2.28) связаны некоторой зависимостью, которую мы будем детализировать в дальнейшем. А именно, пусть

$$
s=s(\tau)>1 \quad \forall \tau \in(0, T], \quad s(0)=1 .
$$

Введем в рассмотрение основную “абсорбционную” энергетическую функцию:

$$
P_{T}(\tau):=I_{\tau}^{T}(s(\tau)):=\int_{\tau}^{T} \int_{\Omega(s(\tau))}\left(d_{0}\left|\nabla_{x} u\right|^{2}+g_{0}(t)|u|^{q+1}\right) d x d t \quad \forall \tau \in[0, T),
$$

где $s(\cdot)$ - определяемая в дальнейшем строго монотонно возрастающая функция из класса $C_{\mathrm{loc}}^{1}(0, T] \cap C^{0}[0, T]$.

Лемма 6. Энергетическая функиия $P_{T}(\cdot)$ из (2.30) является решением следующей задачи Коши для обыкновенного дифференииального неравенства:

$$
\begin{gathered}
P_{T}(\tau) \leqslant c_{1} l_{\tau}(T)^{c(\theta)}\left(-\frac{P_{T}^{\prime}(\tau)}{\varphi_{1}(\tau)}\right)^{1+\lambda_{1}}+c_{0} l(\tau)^{b(\psi)}\left(-\frac{P_{T}^{\prime}(\tau)}{\varphi_{2}(\tau)}\right)^{1+\lambda_{2}} \forall \tau>0, \\
P_{T}(0) \leqslant C F(T),
\end{gathered}
$$

где $F(\cdot)$ из (1.18), константа $C$ из (2.1), постоянные $\theta, \psi$ из (1.22), функиии $b(\cdot), c(\cdot), l_{\tau}(\cdot), l(\cdot)$ и постоянные $c_{0}, c_{1}$ из леммы $5, d_{0}$ из условия (1.6),

$$
\begin{gathered}
\varphi_{1}(\tau)=d_{0} g_{0}(\tau)^{1-\theta} s^{\prime}(\tau), \quad \varphi_{2}(\tau)=g_{0}(\tau)^{1-\psi}, \\
\lambda_{2}:=\frac{(1-\psi)(1-q)}{2-(1-\psi)(1-q)}>\lambda_{1}:=\frac{(1-\theta)(1-q)}{2-(1-\theta)(1-q)}>0 .
\end{gathered}
$$

ДоказАтеЛьство. Легко проверяем, что

$$
\begin{aligned}
0 \geqslant \frac{d P_{T}(\tau)}{d \tau}= & -\int_{\Omega(s(\tau))}\left(d_{0}\left|\nabla_{x} u(\tau, x)\right|^{2}+g_{0}(\tau)|u(\tau, x)|^{q+1}\right) d x \\
& \left.-\left.s^{\prime}(\tau) \int_{\tau}^{T} \int_{\partial_{0} \Omega(s(\tau))}\left(d_{0}\left|\nabla_{x} u\right|^{2}+g_{0}(t) \mid u\right)\right|^{q+1}\right) d \sigma d t .
\end{aligned}
$$

Поэтому, учитывая, что $s^{\prime}(\tau)>0$, выводим из $(2.33)$

$$
\begin{gathered}
E_{\tau}(s(\tau))=\int_{\Omega(s(\tau))}\left(d_{0}\left|\nabla_{x} u(\tau, x)\right|^{2}+g_{0}(\tau)|u(\tau, x)|^{q+1}\right) d x \leqslant-\frac{d P_{T}(\tau)}{d \tau}, \\
J_{\tau}^{T}(s(\tau)) \leqslant \frac{1}{d_{0}} \int_{\tau}^{T} \int_{\partial_{0} \Omega(s(\tau))}\left(d_{0}\left|\nabla_{x} u\right|^{2}+g_{0}(t)|u|^{q+1}\right) d \sigma d t \leqslant-\frac{1}{d_{0} s^{\prime}(\tau)} \frac{d P_{T}(\tau)}{d \tau} .
\end{gathered}
$$

Подставляя эти неравенства в (2.28) и используя определение $(2.30)$, получаем

$$
\begin{aligned}
H_{T}(s(\tau))+P_{T}(\tau) \leqslant & c_{0} l(\tau)^{b(\psi)}\left(-\frac{P_{T}^{\prime}(\tau)}{g_{0}(\tau)^{1-\psi}}\right)^{1+\lambda_{2}} \\
& +c_{1} l_{\tau}(T)^{c(\theta)}\left(-\frac{P_{T}^{\prime}(\tau)}{d_{0} g_{0}(\tau)^{1-\theta} s^{\prime}(\tau)}\right)^{1+\lambda_{1}}
\end{aligned}
$$

что совпадает с дифференциальным неравенством (2.31). Справедливость начального условия (2.32), очевидно, следует из глобальной априорной оценки (2.1). 
Очевидно, что из (2.31) вытекает справедливость неравенства

$$
\begin{aligned}
P_{T}(\tau) \leqslant \max \left\{2 c_{0} l(\tau)^{b(\psi)}\left(-\frac{P_{T}^{\prime}(\tau)}{g_{0}(\tau)^{1-\psi}}\right)^{1+\lambda_{2}},\right. \\
\left.\quad 2 c_{1} l_{\tau}(T)^{c(\theta)}\left(-\frac{P_{T}^{\prime}(\tau)}{d_{0} g_{0}(\tau)^{1-\theta} s^{\prime}(\tau)}\right)^{1+\lambda_{1}}\right\} \forall \tau>0,
\end{aligned}
$$

которое, в свою очередь, легко переписывается в следующем эквивалентном виде:

$$
P_{T}^{\prime}(\tau) \leqslant-\min \left\{h_{2}(\tau) P_{T}(\tau)^{1 /\left(1+\lambda_{2}\right)}, h_{1}(\tau) P_{T}(\tau)^{1 /\left(1+\lambda_{1}\right)}\right\} \quad \forall \tau>0,
$$

где

$$
h_{2}(\tau)=\frac{g_{0}(\tau)^{1-\psi}}{\left(2 c_{0}\right)^{1 /\left(1+\lambda_{2}\right)} l(\tau)^{b(\psi) /\left(1+\lambda_{2}\right)}}, \quad h_{1}(\tau)=\frac{d_{0} g_{0}(\tau)^{1-\theta} s^{\prime}(\tau)}{\left(2 c_{1}\right)^{1 /\left(1+\lambda_{1}\right)} l_{\tau}(T)^{c(\theta) /\left(1+\lambda_{1}\right)}} .
$$

СлеДСтвиЕ 4. Энергетическая функиия $P_{T}(\cdot)$ произвольного решения и задачи (1.1)-(1.4) является решением задачи Коши (2.35), (2.32).

2.2. Доказательство теоремы 1. Ясно, что для доказательства теоремы 1 достаточно показать, что для любого $T>0$ существует $\tau(T): 0<$ $\tau(T)<T$, такое, что $P_{T}(\tau(T))=0$. С этой целью рассмотрим следующую вспомогательную задачу Коши:

$$
\begin{gathered}
P_{T}^{\prime}(\tau)=-\min \left\{\bar{h}_{2}(\tau) \bar{P}_{T}(\tau)^{1 /\left(1+\lambda_{2}\right)}, \bar{h}_{1}(\tau) \bar{P}_{T}(\tau)^{1 /\left(1+\lambda_{1}\right)}\right\} \quad \forall \tau>0, \\
\bar{P}_{T}(0)=C F(T),
\end{gathered}
$$

где

$$
\begin{gathered}
\bar{h}_{2}(\tau)=c_{9} g_{0}(\tau)^{1-\psi}, \quad c_{9}=\left(2 c_{0}\right)^{-1 /\left(1+\lambda_{2}\right)}\left(c_{(n)} L_{1}^{n}\right)^{-b(\psi) /\left(1+\lambda_{2}\right)}, \\
\bar{h}_{1}(\tau)=c_{10} g_{0}(\tau)^{1-\theta} s^{\prime}(\tau), \quad c_{10}=d_{0}\left(2 c_{1}\right)^{-1 /\left(1+\lambda_{1}\right)}\left(c_{(n)} L_{1}^{n}\right)^{-c(\theta) /\left(1+\lambda_{1}\right)},
\end{gathered}
$$

$L_{1}$ из (2.12), остальные параметры те же, что и в системе (2.35), (2.32) (см. лемму 6). Сравнивая задачи (2.35), (2.32) и (2.36), (2.37) и учитывая, что $\bar{h}_{2}(\tau) \leqslant h_{2}(\tau)$ и $\bar{h}_{1}(\tau) \leqslant h_{1}(\tau)$ для любого $\tau>0$, получаем, что произвольные решения этих задач удовлетворяют соотношению

$$
P_{T}(\tau) \leqslant \bar{P}_{T}(\tau) \quad \forall \tau>0 .
$$

Поэтому мы сосредоточимся на изучении поведения решения $\bar{P}_{T}(\cdot)$. Очевидно, что решение задачи Коши (2.36), (2.37) существенно зависит от выбора функции $s(\cdot)$, определяющей структуру функции $\bar{h}_{1}(\cdot)$. Учитывая эту зависимость, решение $\bar{P}_{T}(\cdot)$ задачи $(2.36),(2.37)$ будем также обозначать как $\bar{P}_{T, s(\cdot)}(\cdot)$. Предположим теперь, что при некоторой допустимой функции $s(\cdot)$ для произвольного $T$ : $0<T<T_{0}=$ const, найдется $\delta=\delta(T)>0$ такое, что соответствующее решение $\bar{P}_{T, s(\cdot)}(\cdot)$ обладает свойством

$$
\bar{P}_{T, s(T-\delta)}(T-\delta) \leqslant 0 \text {. }
$$

Тогда, очевидно, имеет место сильная локализация решения, а также следующая оценка радиуса компактификации носителя решения $u$ :

$$
\zeta(T) \leqslant s(T) \quad \forall T<T_{0}
$$


Поэтому достаточным условием появления свойства локализации является нахождение оптимальной функции $s(\cdot)$, обеспечивающей неравенство $(2.38)$ при произвольном $T<T_{0}$ со сколь угодно малым $\delta=\delta(T)>0$.

Отметим также, что $1-\psi>1-\theta, g_{0}(\tau) \rightarrow 0$ при $\tau \rightarrow 0$, а класс допустимых функций $s(\cdot)$ будет состоять из функций таких, что $s^{\prime}(\tau) \geqslant c>0$ для любого $\tau \geqslant 0$. Поэтому для коэффициентов $\bar{h}_{2}(\cdot), \bar{h}_{1}(\cdot)$ уравнения $(2.36)$, очевидно, выполняется соотношение

$$
\bar{h}_{2}(\tau) \cdot\left(\bar{h}_{1}(\tau)\right)^{-1} \rightarrow 0 \quad \text { при } \tau \rightarrow 0 .
$$

Следовательно, уравнение (2.36) по крайней мере при достаточно малых $\tau>0$ совпадает с

$$
\bar{P}_{T}^{\prime}(\tau)=-\bar{h}_{2}(\tau) \bar{P}_{T}(\tau)^{1 /\left(1+\lambda_{2}\right)} .
$$

Решением задачи $(2.41),(2.37)$ является

$$
\bar{P}_{T}^{(2)}(\tau)=\left[(C F(T))^{\lambda_{2} /\left(1+\lambda_{2}\right)}-\frac{\lambda_{2}}{1+\lambda_{2}} \int_{0}^{\tau} \bar{h}_{2}(t) d t\right]^{\left(1+\lambda_{2}\right) / \lambda_{2}} .
$$

Понятно, что при некотором значении $\tau=\tau_{0}>0$ решение $(2.42)$ задачи $(2.36)$, (2.37) "переключается" с режима (2.41) на режим, задаваемый уравнением

$$
\bar{P}_{T}^{\prime}(\tau)=-\bar{h}_{1}(\tau) \bar{P}_{T}(\tau)^{1 /\left(1+\lambda_{1}\right)} \quad \forall \tau>\tau_{0},
$$

т.e.

$$
\bar{P}_{T}^{(1)}(\tau)=\left[\left(\bar{P}_{T}^{(2)}\left(\tau_{0}\right)\right)^{\lambda_{1} /\left(1+\lambda_{1}\right)}-\frac{\lambda_{1}}{1+\lambda_{1}} \int_{\tau_{0}}^{\tau} \bar{h}_{1}(t) d t\right]^{\left(1+\lambda_{1}\right) / \lambda_{1}} .
$$

Причем для точки "переключения" $\tau_{0}$ имеем уравнение

$$
\bar{h}_{1}\left(\tau_{0}\right) \bar{P}_{T}^{(2)}\left(\tau_{0}\right)^{1 /\left(1+\lambda_{1}\right)}=\bar{h}_{2}\left(\tau_{0}\right) \bar{P}_{T}^{(2)}\left(\tau_{0}\right)^{1 /\left(1+\lambda_{2}\right)},
$$

из которого следует справедливость равенства

$$
\bar{P}_{T}^{(2)}\left(\tau_{0}\right)^{\left(\lambda_{2}-\lambda_{1}\right) /\left(\left(1+\lambda_{1}\right)\left(1+\lambda_{2}\right)\right)}=\bar{h}_{2}\left(\tau_{0}\right)\left(\bar{h}_{1}\left(\tau_{0}\right)\right)^{-1}=c_{11} g_{0}^{\theta-\psi}\left(\tau_{0}\right)\left(s^{\prime}\left(\tau_{0}\right)\right)^{-1},
$$

где $c_{11}=c_{9} c_{10}^{-1}$. Будем подбирать функцию $s(\cdot)$ из класса вогнутых функций, поэтому правая часть равенства (2.45) является растущей функцией $\tau_{0}$, а левая часть этого равенства, очевидно, является убывающей функцией $\tau_{0}$. Поэтому ясно, что обязательно существует, причем единственная, точка $\tau_{0}$, для которой выполняется равенство (2.45). С учетом (2.42) из равенства (2.45) выводим уравнение для определения точки $\tau_{0}=\tau_{0}(T)$ :

$$
(C F(T))^{\lambda_{2} /\left(1+\lambda_{2}\right)}-\frac{c_{9} \lambda_{2}}{1+\lambda_{2}} \int_{0}^{\tau_{0}} g_{0}(t)^{1-\psi} d t=\left(\frac{c_{11} g_{0}\left(\tau_{0}\right)^{\theta-\psi}}{s^{\prime}\left(\tau_{0}\right)}\right)^{\lambda_{2}\left(1+\lambda_{1}\right) /\left(\lambda_{2}-\lambda_{1}\right)} .
$$

Легко проверить, что

$$
\frac{\lambda_{2}\left(1+\lambda_{1}\right)}{\lambda_{2}-\lambda_{1}}=\frac{1-\psi}{\theta-\psi}=2 .
$$

Поэтому уравнение (2.46) можно переписать в виде

$$
(C F(T))^{(1-\psi)(1-q) / 2}-\frac{c_{9} \lambda_{2}}{\left(1+\lambda_{2}\right)} \int_{0}^{\tau_{0}} g_{0}(t)^{1-\psi} d t=c_{12} g_{0}\left(\tau_{0}\right)^{1-\psi}\left(s^{\prime}\left(\tau_{0}\right)\right)^{-2},
$$


где $c_{12}=c_{11}^{\lambda_{2}\left(1+\lambda_{1}\right) /\left(\lambda_{2}-\lambda_{1}\right)}=c_{11}^{2}$. Используя полученное в $(2.42),(2.44)$ явное представление решения $\bar{P}_{T}(\cdot)=\bar{P}_{T, s(\cdot)}(\cdot)$ задачи $(2.36),(2.37)$, условие $(2.38)$, достаточное для локализации рассматриваемого решения $u$ исходной задачи, может быть переписано в виде

$$
\begin{gathered}
{\left[(C F(T))^{\lambda_{2} /\left(1+\lambda_{2}\right)}-\frac{\lambda_{2}}{1+\lambda_{2}} \int_{0}^{\tau_{0}} \bar{h}_{2}(t) d t\right]^{\lambda_{1}\left(1+\lambda_{2}\right) /\left(\lambda_{2}\left(1+\lambda_{1}\right)\right)}} \\
-\frac{\lambda_{1}}{1+\lambda_{1}} \int_{\tau_{0}}^{T-\delta} \bar{h}_{1}(t) d t \leqslant 0, \quad \delta>0,
\end{gathered}
$$

или

$$
\begin{array}{ll}
(C F(T))^{\lambda_{2} /\left(1+\lambda_{2}\right)}-\frac{\lambda_{2}}{1+\lambda_{2}} \int_{0}^{\tau_{0}} \bar{h}_{2}(t) d t & \\
\quad<\left(\frac{\lambda_{1}}{1+\lambda_{1}} \int_{\tau_{0}}^{T} \bar{h}_{1}(t) d t\right)^{\lambda_{2}\left(1+\lambda_{1}\right) /\left(\lambda_{1}\left(1+\lambda_{2}\right)\right)} & \forall T<T_{0} .
\end{array}
$$

Наконец, учитывая, что

$$
\begin{gathered}
\frac{\lambda_{2}}{1+\lambda_{2}}=\frac{(1-\psi)(1-q)}{2}, \quad \frac{\lambda_{1}}{1+\lambda_{1}}=\frac{(1-\theta)(1-q)}{2}, \\
\frac{\lambda_{1}\left(1+\lambda_{2}\right)}{\lambda_{2}\left(1+\lambda_{1}\right)}=\frac{1-\theta}{1-\psi}=\frac{1}{2}
\end{gathered}
$$

перепишем (2.48) следующим образом:

$$
\begin{aligned}
& (C F(T))^{(1-\psi)(1-q) / 2} \\
& \quad<c_{13}\left(\int_{\tau_{0}}^{T} g_{0}(t)^{1-\theta} s^{\prime}(t) d t\right)^{2}+c_{14} \int_{0}^{\tau_{0}} g_{0}(t)^{2(1-\theta)} d t \quad \forall T<T_{0},
\end{aligned}
$$

где

$c_{13}=\left(\frac{c_{10} \lambda_{1}}{\left(1+\lambda_{1}\right)}\right)^{2}=\left(\frac{c_{10}(1-\theta)(1-q)}{2}\right)^{2}, \quad c_{14}=\frac{c_{9} \lambda_{2}}{1+\lambda_{2}}=\frac{c_{9}(1-\psi)(1-q)}{2}$.

ЗАмЕчАниЕ 6. Фактически условие (2.49) (где $\tau_{0}$ из $(2.45)$ ) определяет условие подчиненности функции $F$, моделирующей граничный режим, миноранте $g_{0}$. Но это условие является трудно проверяемым для произвольных выпуклых функций $s(\cdot)$, удовлетворяющих (2.29). Поэтому мы получим более простые достаточные условия, соответствующие специальному выбору $s(\cdot)$, а именно положим $s(t)=M t+1$, где $M=$ const $>0$ - произвольный параметр.

Для функции $s(\cdot)$ из замечания 6 достаточное для выполнения условия (2.38) неравенство (2.49) переписывается в виде (1.20) с

$$
S=M\left(\frac{c_{13}}{C^{(1-\psi)(1-q) / 2}}\right)^{1 / 2}, \quad D_{1}=\bar{D}_{1}:=\frac{c_{14}}{C^{(1-\psi)(1-q) / 2}} .
$$

Это последнее зависящее от $L_{1}$ значение $\bar{D}_{1}$ и будем полагать начальным приближением того значения $D_{1}$, которое фигурирует в формулировке теоремы 1 
и которое мы вычислим ниже. Далее, если $S=S(T)$ определяется условием (1.20) при $t=T$, то получаем выполнение соотношения (2.49) с

$$
s(t)=1+t S\left(\frac{c_{13}}{C^{(1-\psi)(1-q) / 2}}\right)^{-1 / 2},
$$

а значит, в силу (2.39)

$$
\begin{gathered}
\zeta(T) \leqslant s(T)=1+c_{15} S T:=1+c_{15} S(T) T \quad \forall T<T_{0}, \\
c_{15}=\left(\frac{c_{13}}{C^{(1-\psi)(1-q) / 2}}\right)^{-1 / 2} .
\end{gathered}
$$

Отсюда в силу условия (1.21) вытекает строгая локализация решения $u$. Обозначим это решение $u$ задачи (1.1)-(1.4), соответствующее области $\Omega=\Omega_{1}$ из (1.1) с $\operatorname{diam} \Omega_{1}=L_{1}$, через $u_{1}$. Зафиксируем произвольное $L_{2}: L_{1}<L_{2}<\infty$, и введем в рассмотрение произвольную ограниченную область с липшицевой границей $\partial \Omega_{2}=\partial_{0} \Omega_{2} \cup \partial_{1} \Omega_{2}$ структуры (1.1) такую, что

$$
\Omega_{1} \cup \partial_{1} \Omega_{1} \subset \Omega_{2}, \quad \operatorname{diam} \Omega_{2}=L_{2} .
$$

Через $u_{2}$ обозначим решение задачи (1.1)-(1.4) в области $\Omega=\Omega_{2}$. В силу $(2.50)$ при $T=T_{1}$, где $T_{1}$ определяется соотношением

$$
1+c_{15} T_{1} S\left(T_{1}\right)=l, \quad l \text { из }(1.1),
$$

функция $U_{1}$, являющаяся продолжением нулем функции $u_{1}$ в дополнение ее носителя, является также решением задачи (1.1)-(1.4) в случае $\Omega=\Omega_{2}$ и в силу единственности решения $u_{2}$ получаем, что $u_{1}(t, x)=u_{2}(t, x)$ для любого $t<T_{1}$. Следовательно, утверждение теоремы 1 с $T=T_{1}$ справедливо для области сколь угодно большого диаметра $L_{2}$ при выполнении условия $(1.20)$ с $0<D_{1}=$ $\bar{D}_{1}\left(n, q, d_{0}, d_{1}, 2 l\right)=$ const $<\infty$, где $l($ см. (1.1)) - единое, не зависящее от diam $\Omega$ число.

2.3. Доказательство следствия 1. В силу неравенства Гёльдера

$$
\int_{0}^{\tau} g_{0}(t)^{2(1-\theta)} d t \geqslant \tau^{-1}\left(\int_{0}^{\tau} g_{0}(t)^{1-\theta} d t\right)^{2},
$$

поэтому для функции $G$ из (1.20) справедливы следующие оценки:

$$
\begin{aligned}
G(S, t, \tau) & \geqslant S^{2}\left(\int_{\tau}^{t} g_{0}(t)^{1-\theta} d t\right)^{2}+\frac{D_{1}}{\tau}\left(\int_{0}^{\tau} g_{0}(t)^{1-\theta} d t\right)^{2} \\
& \geqslant \frac{D_{1} t^{-1} S^{2}}{D_{1} t^{-1}+S^{2}}\left(\int_{0}^{t} g_{0}(t)^{1-\theta} d t\right)^{2} \quad \forall \tau \in[0, t] .
\end{aligned}
$$

Очевидно, что

$$
\frac{D_{1} t^{-1} S^{2}}{D_{1} t^{-1}+S^{2}}=\frac{D_{1}}{t}\left(1-\frac{D_{1} t^{-1}}{D_{1} t^{-1}+S^{2}}\right)=\frac{D_{1}}{2 t},
$$

если $S^{2}:=D_{1} t^{-1}=S^{2}(t)$. Следовательно, из условия (1.24) вытекает выполнение условия $(1.20)$ с $S(t)=\left(D_{1} t^{-1}\right)^{1 / 2}$. Для этой функции выполняется (1.21), а значит, справедлива оценка (1.23) и, как следствие, (1.25). 
2.4. Доказательство следствия 2. Легко проверить, что

$$
\int_{0}^{\tau} g_{0, \beta}(t) d t \approx \frac{\tau^{\beta+1}}{\omega_{0} \beta} g_{0, \beta}(\tau) \quad \text { при } \quad \tau \rightarrow 0 \quad \forall \beta>0 .
$$

Действительно,

$$
\begin{aligned}
\frac{d}{d \tau}\left(\frac{\tau^{\beta+1} \exp \left(-\omega_{0} / \tau^{\beta}\right)}{\omega_{0} \beta}\right) & =\frac{\tau^{\beta} \exp \left(-\omega_{0} / \tau^{\beta}\right)}{\omega_{0} \beta}\left[(\beta+1)+\frac{\omega_{0} \beta}{\tau^{\beta}}\right] \\
& \approx \exp \left(-\frac{\omega_{0}}{\tau^{\beta}}\right) \quad \text { при } \tau \rightarrow 0 .
\end{aligned}
$$

Интегрируя (2.56), получаем (2.55).

Из соотношения (2.55) теперь несложно получить

$$
\int_{0}^{\tau} g_{0, \beta}^{2(1-\theta)}(t) d t \approx \frac{\omega_{0} \beta(1-\theta)}{2 \tau^{\beta+1}}\left(\int_{0}^{\tau} g_{0, \beta}^{1-\theta}(t) d t\right)^{2} \quad \text { при } \tau \rightarrow 0 .
$$

С учетом (2.57) оцениваем функцию $G$ из (1.20):

$$
\begin{aligned}
G(S, t, \tau) & \geqslant S^{2}\left(\int_{\tau}^{t} g_{0, \beta}^{1-\theta}(t) d t\right)^{2}+\frac{D_{2}}{\tau^{1+\beta}}\left(\int_{0}^{\tau} g_{0, \beta}^{1-\theta}(t) d t\right)^{2} \\
& \geqslant \frac{D_{2} t^{-1-\beta} S^{2}}{D_{2} t^{-1-\beta}+S^{2}}\left(\int_{0}^{t} g_{0, \beta}^{1-\theta}(t) d t\right)^{2} \quad \forall \tau \in[0, t],
\end{aligned}
$$

где $D_{2}=2^{-1} D_{1} \beta \omega_{0}(1-\theta)$. Имеем также

$$
\frac{D_{2} t^{-1-\beta} S^{2}}{D_{2} t^{-1-\beta}+S^{2}}=\frac{D_{2}}{t^{1+\beta}}\left(1-\frac{D_{2} t^{-1-\beta}}{D_{2} t^{-1-\beta}+S^{2}}\right)=\frac{D_{2}}{2 t^{1+\beta}},
$$

если $S^{2}:=D_{2} t^{-1-\beta}=S^{2}(t)$. Из (2.58), (2.59) следует справедливость доказываемого утверждения.

2.5. Доказательство следствия 3. В случае $\beta \geqslant 1$ снова воспользуемся оценкой (2.58) для функции $G$ из (1.20). Но теперь положим

$$
S=S(t):=(1+\delta) \mu(t) t^{-1}, \quad \delta=\text { const }>0,
$$

где $\mu(\cdot)$ из (1.30). При этом имеем

$$
\frac{D_{2} t^{-1-\beta} S^{2}}{D_{2} t^{-1-\beta}+S^{2}}=\frac{(1+\delta)^{2} D_{2} \mu^{2}(t)}{D_{2} t^{2}+(1+\delta)^{2} \mu^{2}(t) t^{1+\beta}} \geqslant \frac{\mu^{2}(t)}{t^{2}} .
$$

Последнее неравенство обеспечивается за счет подходящего выбора постоянной $\delta$, так как в случае $\beta \geqslant 1 \mu^{2}(t) t^{1+\beta}=o\left(t^{2}\right)$ при $t \rightarrow 0$. Следовательно, из условия (1.30) в силу (2.58) и (2.61) следует выполнение условия (1.20) теоремы 1 с $S=S(t)$ из (2.60). Для этой последней функции выполняется условие (1.21), а значит, и справедлива оценка (1.23), которая в силу (2.60) соответствует доказываемому неравенству (1.31).

2.6. Пример. Найдем достаточное условие сильной локализации в случае абсорбционного потенциала $g_{0}(\cdot)$ из (1.13):

$$
g_{0}(t)=t^{1 / 2} \exp \left(-\frac{1}{t^{2}}\right) \approx t^{-1 / 2} \operatorname{Erfc}\left(\frac{1}{t}\right)=: t^{-1 / 2} \int_{1 / t}^{\infty} \exp \left(-s^{2}\right) d s .
$$


В силу следствия 1 достаточным условием является

$$
\begin{aligned}
F_{0}(t)^{(1-\psi)(1-q) / 2} & <\frac{D_{1}}{2 t}\left(\int_{0}^{t} \tau^{(1-\theta) / 2} \exp \left(-\frac{1-\theta}{\tau^{2}}\right) d \tau\right)^{2} \\
& \approx \frac{D_{1}}{2 t}\left(\frac{t^{(7-\theta) / 2} \exp \left(-(1-\theta) / t^{2}\right)}{2(1-\theta)}\right)^{2}
\end{aligned}
$$

или

$$
F_{0}(t)<\left(\frac{D_{1}}{8(1-\theta)^{2}} t^{6-\theta} \exp \left(-\frac{2(1-\theta)}{t^{2}}\right)\right)^{2 /((1-\psi)(1-q))} .
$$

ЗАмечАниЕ 7. Очевидно, имеется достаточно большой “зазор” между классом сильно локализованных граничных режимов, определяемым теоремой 1 (и, в частности, условием $(2.62))$, и режимом $f_{0}(t)$ из $(1.13)$, для которого гарантированно отсутствует сильная локализация. Поэтому интересной проблемой, на наш взгляд, является описание точной границы, разделяющей сильно локализованные и нелокализованные граничные режимы в случае сильно вырождающихся в смысле (1.30) абсорбционных потенциалов.

\section{§ 3. Ослабленная локализация при произвольных граничных режимах}

В настоящем параграфе проводится доказательство теоремы 2. Предварительно приведем доказательство ряда вспомогательных утверждений.

3.1. В дополнение к введенным в (2.11), (2.30) энергетическим функциям введем еще одно семейство энергетических функций, связанное с рассматриваемым решением $и$ задачи (1.1)-(1.4):

$$
\begin{gathered}
D_{\tau}(s):=\int_{0}^{\tau} \int_{\Omega(s)}\left(d_{0}\left|\nabla_{x} u\right|^{2}+\mu_{\tau}^{2}(s) u^{2}+g_{0}(t)|u|^{q+1}\right) d x d t \quad \forall \tau \in(0, T], \forall s>1, \\
\mu_{\tau}(s):=\frac{s-1}{8 d_{5} \tau}, \quad d_{5}:=\max \left\{1, d_{1}^{2} d_{0}^{-1}\right\}, \quad g_{0}(t)=\inf _{x \in \Omega} g(t, x),
\end{gathered}
$$

$d_{1}, d_{0}$ - постоянные из условий (1.5) и (1.6) соответственно, а $g$ - потенциал из (1.2). В терминах функции $D_{\tau}(\cdot)$ установим дополнительные "диффузионные" энергетические априорные оценки, которые в определенных ситуациях более точно описывают асимптотические свойства решения $u$, чем установленные ранее "абсорбционные" оценки. Эти "диффузионные" оценки являются оценками типа принципа Сен-Венана в теории упругости, и их вывод в основном соответствует технике, использованной в работе [7]. Поэтому мы приведем лишь новые элементы доказательства, связанные с тем, что, в отличие от [7], мы исследуем поведение решения не на бесконечности, а в окрестности множества $\{|x|=1, t=0\}$.

Лемма 7. Пусть и - произвольное энергетическое решение рассматриваемой задачи (1.1)-(1.4). Тогда при любых $\tau \in(0, T)$ имеет место соотношение $D_{\tau}\left(s_{2}\right) \leqslant \exp \left(-\frac{1}{32 d_{5}^{2} \tau}\left(\frac{\left(s_{2}-1\right)^{2}}{2}-\left(s_{1}-1\right)^{2}\right)\right) D_{\tau}\left(s_{1}\right) \quad \forall s_{2}>s_{1} \geqslant 1+4 d_{5} \sqrt{2 \tau}$,

где функиия $D_{\tau}(\cdot)$ и постоянная $d_{5}-$ из (3.1). 
ЗАмЕЧАниЕ 8. Оценка (3.2) является содержательной только при

$$
\frac{\left(s_{2}-1\right)^{2}}{2}-\left(s_{1}-1\right)^{2}>0
$$

т.е. при $s_{2}-1>\sqrt{2}\left(s_{1}-1\right)$.

ДокАЗАТЕЛЬСтво ЛЕммы 7. В качестве пробной функции в интегральном тождестве (1.17) положим

$$
\xi(t, x)= \begin{cases}u(t, x) e_{\tau, s}(t) \eta_{s, \delta}(|x|) & \text { при } t \in(0, \tau], \quad \tau \leqslant T \\ 0 & \text { при } t \geqslant \tau\end{cases}
$$

где $e_{\tau, s}(t)=\exp \left(-\mu_{\tau}^{2}(s) t\right), \mu_{\tau}(\cdot)-$ из $(3.1), \eta_{s, \delta}(|x|)-$ срезающая функция из (2.10). Применяя стандартно формулу интегрирования по частям и переходя к пределу при $\delta \rightarrow 0$, приходим при почти всех $s>1$ к равенству

$$
\begin{gathered}
\frac{1}{2} \int_{\Omega(s)} u(\tau, x)^{2} e_{\tau, s}(t) d x+\int_{0}^{\tau} \int_{\Omega(s)} \sum_{i=1}^{n} a_{i}\left(t, x, u, \nabla_{x} u\right) u_{x_{i}} e_{\tau, s}(t) d x d t \\
\quad+\int_{0}^{\tau} \int_{\Omega(s)}\left(\mu_{\tau}^{2}(s) u^{2}+g(t, x)|u|^{q+1}\right) e_{\tau, s}(t) d x d t \\
=\int_{0}^{\tau} \int_{\partial_{0} \Omega(s)} \sum_{i=1}^{n} a_{i}\left(t, x, u, \nabla_{x} u\right) u \nu_{i} e_{\tau, s}(t) d \sigma d t
\end{gathered}
$$

В силу условия (1.5) и неравенства Юнга имеем

$$
\begin{aligned}
& \int_{0}^{\tau} \int_{\partial_{0} \Omega(s)} \sum_{i=1}^{n} a_{i}\left(t, x, u, \nabla_{x} u\right) u \nu_{i} e_{\tau, s}(t) d \sigma d t \\
& \quad \leqslant \frac{1}{\mu_{\tau}(s)} \int_{0}^{\tau} \int_{\partial_{0} \Omega(s)}\left(d_{1}^{2}\left|\nabla_{x} u\right|^{2}+\mu_{\tau}^{2}(s) u^{2}\right) e_{\tau, s}(t) d \sigma d t .
\end{aligned}
$$

Теперь из равенства (3.4) в силу структурного условия (1.6) и оценки (3.5) следует

$$
\begin{gathered}
\frac{1}{2} \int_{\Omega(s)} u(\tau, x)^{2} e_{\tau, s}(t) d x+\int_{0}^{\tau} \int_{\Omega(s)}\left(d_{0}\left|\nabla_{x} u\right|^{2}+\mu_{\tau}^{2}(s) u^{2}+g_{0}(t)|u|^{q+1}\right) e_{\tau, s}(t) d x d t \\
\leqslant \frac{1}{\mu_{\tau}(s)} \int_{0}^{\tau} \int_{\partial_{0} \Omega(s)}\left(d_{1}^{2}\left|\nabla_{x} u\right|^{2}+\mu_{\tau}^{2}(s) u^{2}\right) e_{\tau, s}(t) d \sigma d t
\end{gathered}
$$

Введем параметрическое семейство функций аргумента $s$ :

$$
A_{\tau}(s):=\int_{0}^{\tau} \int_{\Omega(s)}\left(d_{0}\left|\nabla_{x} u\right|^{2}+\mu_{\tau}^{2}(s) u^{2}+g_{0}(t)|u|^{q+1}\right) e_{\tau, s}(t) d x d t \quad \forall s>1 .
$$


Заметим, что

$$
\begin{aligned}
\frac{d A_{\tau}(s)}{d s}= & -\int_{0}^{\tau} \int_{\partial_{0} \Omega(s)}\left(d_{0}\left|\nabla_{x} u\right|^{2}+\mu_{\tau}^{2}(s) u^{2}+g_{0}(t)|u|^{q+1}\right) e_{\tau, s}(t) d \sigma d t \\
& -2 \mu_{\tau}(s) \mu_{\tau}^{\prime}(s) \int_{0}^{\tau} \int_{\Omega(s)} t\left(d_{0}\left|\nabla_{x} u\right|^{2}+\mu_{\tau}^{2}(s) u^{2}+g_{0}(t)|u|^{q+1}\right) e_{\tau, s}(t) d x d t \\
& +2 \mu_{\tau}(s) \mu_{\tau}^{\prime}(s) \int_{0}^{\tau} \int_{\Omega(s)} u^{2} e_{\tau, s}(t) d x d t
\end{aligned}
$$

откуда с учетом того, что $\mu_{\tau}^{\prime}(s) \geqslant 0$, следует

$$
\begin{aligned}
& \frac{1}{\mu_{\tau}(s)} \int_{0}^{\tau} \int_{\partial_{0} \Omega(s)}\left(d_{1}^{2}\left|\nabla_{x} u\right|^{2}+\mu_{\tau}^{2}(s) u^{2}\right) e_{\tau, s}(t) d \sigma d t \\
& \quad \leqslant \frac{d_{5}}{\mu_{\tau}(s)} \int_{0}^{\tau} \int_{\partial_{0} \Omega(s)}\left(d_{0}\left|\nabla_{x} u\right|^{2}+\mu_{\tau}^{2}(s) u^{2}+g_{0}(t)|u|^{q+1}\right) e_{\tau, s}(t) d \sigma d t \\
& \quad \leqslant-\frac{d_{5}}{\mu_{\tau}(s)} \frac{d A_{\tau}(s)}{d s}+\frac{2 d_{5} \mu_{\tau}^{\prime}(s)}{\mu_{\tau}^{2}(s)} A_{\tau}(s) .
\end{aligned}
$$

Используя неравенство (3.7) для оценивания сверху правой части соотношения (3.6), после приведения подобных членов получаем

$$
\left(1-2 d_{5} \mu_{\tau}^{\prime}(s) \mu_{\tau}^{-2}(s)\right) A_{\tau}(s) \leqslant-\frac{d_{5}}{\mu_{\tau}(s)} \frac{d A_{\tau}(s)}{d s} .
$$

Легко проверить, что для выбранной нами функции $\mu_{\tau}(\cdot)$ из (3.1) выполняется соотношение

$$
1-2 d_{5} \mu_{\tau}^{\prime}(s) \mu_{\tau}^{-2}(s) \geqslant \frac{1}{2} \quad \forall s \geqslant 1+4 d_{5} \sqrt{2 \tau} .
$$

Таким образом, из (3.8) и (3.9) вытекает, что энергетическая функция $A_{\tau}(\cdot)$ удовлетворяет следующему дифференциальному неравенству:

$$
\frac{1}{2} A_{\tau}(s) \leqslant-\frac{d_{5}}{\mu_{\tau}(s)} \frac{d A_{\tau}(s)}{d s} .
$$

Проинтегрировав (3.10) по $s \in\left(s_{1}, s_{2}\right), s_{1} \geqslant 1+4 d_{5} \sqrt{2 \tau}$, получим

$$
A_{\tau}\left(s_{2}\right) \leqslant A_{\tau}\left(s_{1}\right) \exp \left(-\frac{1}{2 d_{5}} \int_{s_{1}}^{s_{2}} \mu_{\tau}(s) d s\right) \quad \forall \tau \in(0, T] .
$$

Отсюда в результате простых вычислений следует

$$
\begin{gathered}
\exp \left(-\frac{\left(s_{2}-1\right)^{2}}{64 d_{5}^{2} \tau}\right) \int_{0}^{\tau} \int_{\Omega\left(s_{2}\right)}\left(d_{0}\left|\nabla_{x} u\right|^{2}+\left(\frac{s_{2}-1}{8 d_{5} \tau}\right)^{2} u^{2}+g_{0}(t)|u|^{q+1}\right) d x d t \\
\leqslant \exp \left(-\frac{\left\{\left(s_{2}-1\right)^{2}-\left(s_{1}-1\right)^{2}\right\}}{32 d_{5}^{2} \tau}\right) \\
\times \int_{0}^{\tau} \int_{\Omega\left(s_{1}\right)}\left(d_{0}\left|\nabla_{x} u\right|^{2}+\left(\frac{s_{1}-1}{8 d_{5} \tau}\right)^{2} u^{2}+g_{0}(t)|u|^{q+1}\right) d x d t \\
\forall s_{2}>s_{1} \geqslant 1+4 d_{5} \sqrt{2 \tau},
\end{gathered}
$$

что соответствует доказываемой оценке (3.2). 
ЗАмЕчАниЕ 9. Легко видеть, что из глобальной априорной оценки (2.1) вытекает также следующее неравенство:

$$
\begin{gathered}
\int_{0}^{\tau} \int_{\Omega}\left(d_{0}\left|\nabla_{x} u\right|^{2}+\frac{\lambda}{\tau} u^{2}+g_{0}(t)|u|^{q+1}\right) d x d t \leqslant C \max \{1, \lambda\} F(\tau) \\
\forall \tau \leqslant T, \quad \forall \lambda>0,
\end{gathered}
$$

где $C$ - постоянная из $(2.1)$, а $F(\cdot)$ - функция из (1.18).

Лемма 8. Для энергетической функиии $D_{\tau}(\cdot)$, определенной в $(3.1)$, справедлива оценка

$$
D_{\tau}(s) \leqslant C_{1} \exp \left(-\frac{(s-1)^{2}}{64 d_{5}^{2} \tau}\right) F(\tau) \quad \forall \tau>0, \quad \forall s>\bar{s}(\tau)=1+4 d_{5} \sqrt{2 \tau},
$$

где $C_{1}=C \exp (1), C$ - постоянная из (2.1), а $F(\cdot)$ - функиия из (1.18).

ДокАзАтЕЛЬСтво. Учитывая, что $\mu_{\tau}^{2}(\bar{s}(\tau))=1 /(2 \tau)$, выводим из неравенства (3.11) замечания 9 при $\lambda=1 / 2$ оценку

$$
D_{\tau}(\bar{s}(\tau))=\int_{0}^{\tau} \int_{\Omega(\bar{s}(\tau))}\left(d_{0}\left|\nabla_{x} u\right|^{2}+\frac{u(t, x)^{2}}{2 \tau}+g_{0}(t)|u(t, x)|^{q+1}\right) d x d t \leqslant C F(\tau)
$$

для любого $\tau>0, C$ - постоянная из (2.1). Теперь из соотношения (3.2) при $s=s_{2}>\bar{s}(\tau)$ и $s_{1}=\bar{s}(\tau)$ выводим с учетом (3.13)

$$
\begin{aligned}
D_{\tau}\left(s_{2}\right) & \leqslant \exp \left(-\frac{1}{32 d_{5}^{2} \tau}\left(\frac{(s-1)^{2}}{2}-(\bar{s}-1)^{2}\right)\right) D_{\tau}(\bar{s}(\tau)) \\
& \leqslant \exp \left(-\frac{(s-1)^{2}}{64 d_{5}^{2} \tau}\right) \exp \left(\frac{(\bar{s}-1)^{2}}{32 d_{5}^{2} \tau}\right) D_{\tau}(\bar{s}(\tau)) \\
& \leqslant \exp \left(-\frac{(s-1)^{2}}{64 d_{5}^{2} \tau}\right) \exp (1) C F(\tau)
\end{aligned}
$$

что и соответствует необходимой оценке (3.12).

3.2. Дальнейшее доказательство теоремы 2, т.е. описание носителя решения в случае, когда возможен конечный или даже бесконечный начальный скачок, проводится с использованием энергетического метода исследования свойства мгновенной компатификации носителей решений параболических уравнений с невырождающимся абсорбционным потенциалом (см. [11], [12]). Проведем теперь некоторую "локализацию" оценок из п. 2.1, которые привели к основному энергетическому соотношению (2.28). Зафиксируем произвольное число $R: 0<R<L_{1}-1$, и точку $x_{R} \in \Omega$ : $\left|x_{R}\right|=R+1$. Наряду с семейством подобластей $\Omega(s)$ из $(2.8)$ введем семейство равномерно ограниченных подобластей

$$
\Omega_{R}(s):=\left\{x \in \Omega:\left|x-x_{R}\right|<1-s\right\} \quad \forall s \in[0,1), \quad \operatorname{diam} \Omega_{R}(s) \leqslant 2,
$$

а также связанные с ними семейства аналогичных (2.11) энергетических функций 


$$
\begin{gathered}
H_{\tau}(s):=H_{R, \tau}(s)\left(:=\int_{\Omega_{R}(s)}|u(\tau, x)|^{2} d x\right), \\
E_{\tau}(s):=E_{R, \tau}(s), \quad I_{\tau}^{b}(s):=I_{R, \tau}^{b}(s), \quad J_{\tau}^{b}(s):=J_{R, \tau}^{b}(s) .
\end{gathered}
$$

Очевидно, что для областей $\Omega_{R}(s)$ из (3.15) неравенство (2.17) приобретает вид

$$
\begin{gathered}
\int_{\Omega_{R}(s)}|u(t, x)|^{q+1} d x \leqslant c_{(n)}^{(1-q) / 2}\left(\int_{\Omega_{R}(s)}|u(t, x)|^{2} d x\right)^{(q+1) / 2} \\
\forall s \in[0,1), \quad c_{(n)} \quad \text { из }(2.13),
\end{gathered}
$$

а интерполяционные неравенства (2.15), (2.26) - соответственно вид

$$
\begin{gathered}
\int_{\partial \Omega_{R}(s)}|u(t, x)|^{2} d \sigma \leqslant d_{3}\left(\int_{\Omega_{R}(s)}\left|\nabla_{x} u\right|^{2} d x\right)^{\theta}\left(\int_{\Omega_{R}(s)}|u(t, x)|^{q+1} d x\right)^{2(1-\theta) /(q+1)} \\
+\bar{d}_{3}\left(\int_{\Omega_{R}(s)}|u(t, x)|^{q+1} d x\right)^{2 /(q+1)}:=d_{3} M(\theta)+\bar{d}_{3} M(0) \quad \forall t>0,
\end{gathered}
$$

где $\theta$ из $(1.22), d_{3}=d_{3}(n, q)=$ const, $\bar{d}_{3}=\bar{d}_{3}(n, q)=$ const,

$$
\begin{gathered}
\int_{\partial \Omega_{R}(s)}|u(t, x)|^{2} d \sigma \leqslant d_{4}\left(\int_{\Omega_{R}(s)}\left|\nabla_{x} u\right|^{2} d x\right)^{\psi}\left(\int_{\Omega_{R}(s)}|u(t, x)|^{q+1} d x\right)^{2(1-\psi) /(q+1)} \\
+\bar{d}_{3}\left(\int_{\Omega_{R}(s)}|u(t, x)|^{q+1} d x\right)^{2 /(q+1)}:=d_{4} M(\psi)+\bar{d}_{4} M(0) \quad \forall t>0,
\end{gathered}
$$

где $\psi$ из $(1.22), d_{4}=d_{4}(n, q)=\mathrm{const}, \bar{d}_{4}=\bar{d}_{4}(n, q)=$ const. Повторяя теперь доказательства лемм 2-5 с заменой в соответствующих местах неравенств (2.17), $(2.15),(2.26)$ на (3.17)-(3.19), приходим к аналогичному (2.28) соотношению

$$
\begin{aligned}
H_{T}(s)+ & I_{\tau}^{T}(s) \leqslant c_{1} g_{0}(\tau)^{-a(\theta)}\left[J_{\tau}^{T}(s)\right]^{d(\theta)}+c_{2} g_{0}(\tau)^{-a(\psi)} E_{\tau}(s)^{d(\psi)} \\
& +c_{3} g_{0}(\tau)^{-a(0)} E_{\tau}(s)^{d(0)}+c_{4} g_{0}(\tau)^{-a(0)}\left[J_{\tau}^{T}(s)\right]^{d(0)} \quad \forall s \in[0,1), \quad \forall \tau \leqslant T,
\end{aligned}
$$

где $\theta, \psi$ - постоянные из (1.22), а функции $a(\cdot), d(\cdot)$ те же, что и в $(2.28)$. Ориентируясь на определение (2.30) энергетической функции $P_{T}(\cdot)$, введем теперь в рассмотрение следующее двухпараметрическое семейство энергетических функций:

$$
P_{T}(\tau):=P_{R, T}(\tau):=I_{T / 2+\tau}^{T}(\tau) \quad \forall \tau: 0<\tau<\frac{T}{2}, \quad \text { т.e. } s(\tau):=\tau,
$$

где $I_{T / 2+\tau}^{T}(\tau)$ из (3.16). При этом аналогом дифференциального неравенства (2.31) является

$$
\begin{aligned}
& P_{T}(\tau) \leqslant c_{1}\left(-\frac{P_{T}^{\prime}(\tau)}{g_{0}(T / 2)^{1-\theta}}\right)^{1+\lambda_{1}}+c_{2}\left(-\frac{P_{T}^{\prime}(\tau)}{g_{0}(T / 2)^{1-\psi}}\right)^{1+\lambda_{2}}+c_{3}\left(-\frac{P_{T}^{\prime}(\tau)}{g_{0}(T / 2)}\right)^{1+\lambda_{3}} \\
& \forall \tau: 0<\tau<\frac{T}{2},
\end{aligned}
$$


где $\lambda_{i}, i=1,2,-$ постоянные из $(2.31), \lambda_{3}=(1-q) /(1+q)$. Следуя доказательству теоремы 1 , введем аналог вспомогательного неравенства (2.35):

$$
\begin{gathered}
\frac{d}{d \tau} P_{T}(\tau) \leqslant-\min \left\{c_{6} g_{0}\left(\frac{T}{2}\right)^{1-\psi} P_{T}(\tau)^{1 /\left(1+\lambda_{2}\right)}, c_{7} g_{0}\left(\frac{T}{2}\right)^{1-\theta} P_{T}(\tau)^{1 /\left(1+\lambda_{1}\right)},\right. \\
\left.c_{5} g_{0}\left(\frac{T}{2}\right) P_{T}(\tau)^{1 /\left(1+\lambda_{3}\right)}\right\} \quad \forall \tau: 0<\tau<\frac{T}{2} .
\end{gathered}
$$

Соответственно, аналог (2.36) это

$$
\begin{gathered}
\bar{P}_{T}^{\prime}(\tau)=-\min \left\{c_{6} g_{0}\left(\frac{T}{2}\right)^{1-\psi} \bar{P}_{T}(\tau)^{1 /\left(1+\lambda_{2}\right)}, c_{7} g_{0}\left(\frac{T}{2}\right)^{1-\theta} \bar{P}_{T}(\tau)^{1 /\left(1+\lambda_{1}\right)},\right. \\
\left.c_{5} g_{0}\left(\frac{T}{2}\right) \bar{P}_{T}(\tau)^{1 /\left(1+\lambda_{3}\right)}\right\} \quad \forall \tau: 0<\tau<\frac{T}{2} .
\end{gathered}
$$

Кроме того, заметим, что из определения $(3.21)$ следует $P_{T}(0):=P_{R, T}(0):=$ $I_{R, T / 2}^{T}(0) \leqslant D_{T}(R)$, поэтому в силу оценки $(3.12)$ из леммы 8 имеем

$$
P_{T}(0) \leqslant C_{1} \exp \left(-\frac{(R-1)^{2}}{64 d_{5}^{2} T}\right) F(T):=N_{T}(R) F(T):=F_{R}(T) .
$$

3.3. Доказательство теоремы 2 состоит в анализе зависимости локализации решений задачи Коши (3.23), (3.24) (а следовательно, и исходной задачи (1.1)-(1.4)) от параметра $R$. Ясно, что $P_{R, T}(\tau) \leqslant \bar{P}_{R, T}(\tau)$ для любого $\tau>0$. Поэтому вопрос о локализации решений задачи Коши (3.23), (3.24) сводится к проверке того, что при любом $T>0$ существует такое значение $R=R(T)<\infty$, что имеет место аналогичное $(2.38)$ неравенство

$$
\bar{P}_{T}\left(\frac{T}{2}-\delta\right):=\bar{P}_{R, T}\left(\frac{T}{2}-\delta\right) \leqslant 0
$$

со сколь угодно малым $\delta$ : $0<\delta<T / 2$. Отсюда, очевидно, следует ослабленная локализация решения, а также оценка радиуса компактификации носителя решения $u$

$$
\zeta(T) \leqslant R(T)+\frac{T}{2} .
$$

Решениями “укороченных" обыкновенных дифференциальных уравнений

$$
\begin{gathered}
\bar{P}_{T}^{\prime}(\tau)=-c_{7} g_{0}\left(\frac{T}{2}\right)^{1-\theta} \bar{P}_{T}(\tau)^{1 /\left(1+\lambda_{1}\right)}, \quad \bar{P}_{T}^{\prime}(\tau)=-c_{6} g_{0}\left(\frac{T}{2}\right)^{1-\psi} \bar{P}_{T}(\tau)^{1 /\left(1+\lambda_{2}\right)}, \\
\bar{P}_{T}^{\prime}(\tau)=-c_{5} g_{0}\left(\frac{T}{2}\right) \bar{P}_{T}(\tau)^{1 /\left(1+\lambda_{3}\right)} \quad \forall \tau: 0<\tau<\frac{T}{2}
\end{gathered}
$$

с начальным условием (3.24) являются соответственно функции

$$
\begin{aligned}
& \bar{P}_{T, R}^{(1)}(\tau)=F_{R}(T)-\left(\frac{(1-\theta)(1-q)}{2} c_{7} g_{0}\left(\frac{T}{2}\right)^{1-\theta} \tau\right)^{2 /((1-\theta)(1-q))}, \\
& \bar{P}_{T, R}^{(2)}(\tau)=F_{R}(T)-\left(\frac{(1-\psi)(1-q)}{2} c_{6} g_{0}\left(\frac{T}{2}\right)^{1-\psi} \tau\right)^{2 /((1-\psi)(1-q))},
\end{aligned}
$$




$$
\bar{P}_{T, R}^{(3)}(\tau)=F_{R}(T)-\left(\frac{1+q}{2} c_{5} g_{0}\left(\frac{T}{2}\right) \tau\right)^{2 /(1+q)} \quad \forall \tau: 0<\tau<\frac{T}{2} .
$$

В силу того, что $1-\theta=(1-\psi) / 2$ и справедливы неравенства

$$
\frac{2}{(1-\theta)(1-q)}=\frac{4}{(1-\psi)(1-q)}>\frac{2}{(1-\psi)(1-q)}>\frac{2}{1+q},
$$

получаем соотношения

$$
\bar{P}_{T, R}^{(1)}(\tau)>\bar{P}_{T, R}^{(2)}(\tau)>\bar{P}_{T, R}^{(3)}(\tau) \quad \forall \tau: 0<\tau<\frac{T}{2}
$$

при достаточно малых $T$. Из вида уравнения (3.23) теперь легко получаем, что решением задачи Коши (3.23), (3.24) является

$$
\begin{aligned}
\bar{P}_{T, R}(\tau)=F_{R}(T) & \\
& -\left(\frac{(1-\theta)(1-q)}{2} c_{7} g_{0}\left(\frac{T}{2}\right)^{1-\theta} \tau\right)^{2 /((1-\theta)(1-q))} \quad \forall \tau: 0<\tau \leqslant \frac{T}{2} .
\end{aligned}
$$

Условие (3.25) с учетом представления (3.27) приводит к следующему достаточному условию локализации:

$$
F_{R}(T) \leqslant c_{8} g_{0}\left(\frac{T}{2}\right)^{2 /(1-q)} \tau^{2 /((1-\theta)(1-q))} \quad \forall \tau: 0<\tau \leqslant \frac{T}{2},
$$

где $c_{8}:=\left(((1-\theta)(1-q) / 2) c_{7}\right)^{2 /((1-\theta)(1-q))}$. Следовательно, в качестве финального достаточного условия локализации (из (3.28) с учетом определения $F_{R}(T)$ ) получаем

$$
N_{T}(R)<(F(T))^{-1} c_{8} g_{0}\left(\frac{T}{2}\right)^{2 /(1-q)} \tau^{2 /((1-\theta)(1-q))} \quad \forall \tau: 0<\tau \leqslant \frac{T}{2} .
$$

Последнее неравенство в силу определения (3.24) приводит к

$$
R>\bar{R}(T):=1+8 d_{5} T^{1 / 2}\left\{\ln \left(\frac{C_{1} F(T)}{c_{8} g_{0}(T / 2)^{2 /(1-q)}(T / 2)^{2 /((1-\theta)(1-q))}}\right)\right\}^{1 / 2} .
$$

Отметим, что $g_{0}(T / 2)>0$ для любого $T>0$. Следовательно, $\bar{R}(T)<\infty$ для любого $T>0$. Из определения (3.21) энергетической функции $P_{R, T}(\cdot)$ теперь следует, что при выполнении условия (3.29) на значение параметра $R$ в $(3.25)$ выполнено достаточное условие локализации (3.28) при любом $T>0$ и для радиуса локализации $\zeta(\cdot)$ справедлива оценка

$$
\begin{aligned}
\zeta(T) & \leqslant 1+8 d_{5} T^{1 / 2}\left\{\ln \left(\frac{C_{1} F(T)}{c_{8} g_{0}(T / 2)^{2 /(1-q)}(T / 2)^{2 /((1-\theta)(1-q))}}\right)\right\}^{1 / 2}+\frac{T}{2} \\
& :=\zeta_{1}(T)<\infty
\end{aligned}
$$

что и соответствует утверждению теоремы 2. 
ЗАМЕЧАНИЕ 10. $\zeta_{1}(\cdot)$ и есть искомая функция, которая фигурирует в формулировке теоремы 2 .

ЗАмЕЧАнИЕ 11. В силу монотонности функции $\zeta_{1}(\cdot)$ из уравнения $L_{1}=$ $\zeta_{1}\left(t_{0}\right)$ несложно найти $t_{0}=\zeta_{1}^{-1}\left(L_{1}\right)=t_{0}\left(L_{1}\right) \rightarrow 0$ при $L_{1} \rightarrow \infty$, тогда справедлива оценка $\zeta(t) \leqslant \zeta_{1}(t)$ для любого $t \geqslant t_{0}$. В случае $L_{1}=\infty$ имеем оценку $\zeta(t) \leqslant \zeta_{1}(t)<\infty$ для любого $t>0$.

\section{§ 4. Сильная локализация при ограничениях на абсорбционный потенциал}

Для доказательства теоремы 3 , очевидно, достаточно показать, что функция $\zeta_{1}(T)$ из (3.30) обладает следующим свойством:

$$
\zeta_{1}(T) \rightarrow 1 \quad \text { при } T \rightarrow 0 .
$$

Отметим, что в рассмариваемой сейчас ситуации минорантой $g_{0}(\cdot)$ является функция $g_{\omega}(\cdot)$ из (1.33). Поэтому соотношение $(3.29)$ приводит к

$$
\bar{R}(T)=1+8 d_{5}\left\{R_{1}+R_{2}+R_{3}\right\}^{1 / 2},
$$

где $R_{1}=R_{1}(T)=T \ln \left(C_{1} F(T)\right), R_{2}=R_{2}(T)=2 T \ln \left(c_{8} T / 2\right)^{-1} /((1-\theta)(1-q))$, $R_{3}=R_{3}(T)=4 \omega(T / 2) /(1-q)$. В силу монотонности функции $F(\cdot)$ имеем

$$
R_{1}(T) \leqslant T \ln \left(C_{1} F\left(T_{0}\right)\right):=R_{1}^{(0)}(T) \quad \forall T<T_{0} .
$$

Поэтому

$$
R_{1}(T) \leqslant R_{1}^{(0)}(T) \rightarrow 0 \quad \text { при } T \rightarrow 0 .
$$

где $c=2 /((1-\psi)(1-q))$. Очевидно, что

$$
R_{2}(T) \rightarrow 0 \quad \text { при } T \rightarrow 0 .
$$

Ясно также, что из условий на функцию $\omega(\cdot)$ следует:

$$
R_{3}(T) \rightarrow 0 \quad \text { при } T \rightarrow 0 .
$$

Таким образом, из (4.2)-(4.5) вытекает, что

$$
\bar{R}(T) \rightarrow 1 \quad \text { при } T \rightarrow 0 .
$$

Отсюда в силу (3.30) следует сильная локализация рассматриваемого решения $u$ задачи (1.1)-(1.4), причем справедлива следующая оценка для радиуса компактификации:

$$
\zeta(T) \leqslant 1+\frac{T}{2}+8 d_{5}\left\{T \ln \left(C_{1} F(T)\right)+\frac{2 T \ln \left(c_{8} T / 2\right)^{-1}}{(1-\theta)(1-q)}+\frac{4 \omega(T / 2)}{1-q}\right\}^{1 / 2} \forall T<T_{0} .
$$

Статья посвящена светлой памяти Анатолия Сергеевича Калашникова. 


\section{Список литературы}

[1] H.W. Alt, S. Luckhaus, "Quasilinear elliptic-parabolic differential equations", Math. Z., 183:3 (1983), 311-341.

[2] S. Antontsev, J. I. Diaz, S. I. Shmarev, "The support shrinking properties for solutions of quasilinear parabolic equations with strong absorption terms", Ann. Fac. Sci. Toulouse Math. (6), 4:1 (1995), 5-30.

[3] J. I. Diaz, L. Veron, "Laurent Local vanishing properties of solutions of elliptic and parabolic quasilinear equations", Trans. Amer. Math. Soc., 290:2 (1985), 787-814.

[4] А.С. Калашников, "Некоторые вопросы качественной теории нелинейных вырождающихся параболических уравнений второго порядка", УМH, 42:2 (1987), 135-176; англ. пер.: A.S. Kalashnikov, "Some problems of the qualitative theory of non-linear degenerate second-order parabolic equations", Russian Math. Surveys, 42:2 (1987), 169-222.

[5] А. С. Калашников, "О начальном скачке свободной границы в краевой задаче для полулинейного уравнения теплопроводности с поглощением”, УМH, 52:6 (1997), 163-164; англ. пер.: A.S. Kalashnikov, "On the initial jump of the free boundary in a boundary-value problem for the semilinear heat conduction equation with absorption", Russian Math. Surveys, 52:6 (1997), 1300-1301.

[6] С. Н. Антонцев, "О локализации решений нелинейных вырождающихся эллиптических и параболических уравнений", Докл. АН СCCP, 260:6 (1981), 1289-1293; англ. пер.: S. N. Antontsev, "On the localization of solutions of nonlinear degenerate elliptic and parabolic equations", Soviet Math. Dokl., 24:2 (1981), 420-424.

[7] О.А.Олейник, Г. А. Иосифьян, "Аналог принципа Сен-Венана для эллиптического уравнения второго порядка и единственность решений краевых задач в неограниченных областях", УМН, 31:4 (1976), 261-262.

[8] A. Shishkov, L. Veron, "The balance between diffusion and absorption in semilinear parabolic equations", Atti Accad. Naz. Lincei Cl. Sci. Fis. Mat. Natur. Rend. Lincei (9) Mat. Appl., 18:1 (2007), 59-96.

[9] Y. Belaud, A. Shishkov, "Long-time extinction of solutions of some semilinear parabolic equations", J. Differential Equations, 2007, № 1, 64-86.

[10] Ж.-Л. Лионс, Некоторые методы решения нелинейных краевых задач, Мир, М., 1972; пер. с фр.: J.-L. Lions, Quelques méthodes de résolution des problèmes aux limites non linéaires, Dunod, de Gruyter, Paris, 1969.

[11] R. Kersner, A. Shishkov, "Instantaneous shrinking of the support of energy solutions", J. Math. Anal. Appl., 198:3 (1996), 729-750.

[12] А. Е. Шишков, “Мертвые зоны и мгновенная компактификация носителей энергетических решений квазилинейных параболических уравнений произвольного порядка", Матем. сб., 190:12 (1999), 129-156; англ. пер.: А. E. Shishkov, "Dead cores and instantaneous compactification of the supports of energy solutions of quasilinear parabolic equations of arbitrary order", Sb. Math., 190:12 (1999), 1843-1869.

\section{Е. В. Степанова (K. V. Stiepanova)}

Институт прикладной математики и механики

НАН Украины, г. Донецк

E-mail: kitti_dob@rambler.ru

\section{А. Е. Шишков (А. E. Shishkov)}

Институт прикладной математики и механики

НАН Украины, г. Донецк;

Донецкий национальный университет, Украина

E-mail: aeshkv@yahoo.com
Поступила в редакцию 22.03.2012 и 03.08.2012 